\title{
QTL controlling fiber quality traits under salt stress in upland cotton (Gossypium hirsutum L.)
}

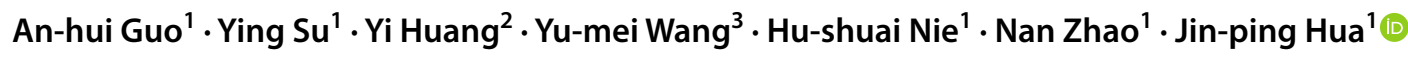

Received: 1 March 2020 / Accepted: 31 October 2020 / Published online: 2 January 2021

(c) The Author(s) 2021

\begin{abstract}
Key message QTL for fiber quality traits under salt stress discerned candidate genes controlling fatty acid metabolism. Abstract Salinity stress seriously affects plant growth and limits agricultural productivity of crop plants. To dissect the genetic basis of response to salinity stress, a recombinant inbred line population was developed to compare fiber quality in upland cotton (Gossypium hirsutum L.) under salt stress and normal conditions. Based on three datasets of (1) salt stress, (2) normal growth, and (3) the difference value between salt stress and normal conditions, 51, 70, and 53 QTL were mapped, respectively. Three QTL for fiber length (FL) ( $q F L-C h r 1-1, q F L-C h r 5-5$, and $q F L-C h r 24-4$ ) were detected under both salt and normal conditions and explained $4.26 \%, 9.38 \%$, and $3.87 \%$ of average phenotypic variation, respectively. Seven genes within intervals of two stable QTL ( $q F L-C h r l-1$ and $q F L-C h r 5-5)$ were highly expressed in lines with extreme long fiber. A total of 35 QTL clusters comprised of 107 QTL were located on 18 chromosomes and exhibited pleiotropic effects. Thereinto, two clusters were responsible for improving five fiber quality traits, and 6 influenced FL and fiber strength (FS). The QTL with positive effect for fiber length exhibited active effects on fatty acid synthesis and elongation, but the ones with negative effect played passive roles on fatty acid degradation under salt stress.
\end{abstract}

\section{Introduction}

Soil salinity, as one of the major abiotic stresses, reduces global agricultural productivity due to the harmful effects on plant growth. Two main approaches can be used to produce salt-tolerant crops: (i) exploitation of natural genetic variations by direct selection or mapping quantitative trait loci

Communicated by Peter Langridge.

Supplementary Information The online version of this article (https://doi.org/10.1007/s00122-020-03721-x) contains supplementary material, which is available to authorized users.

Jin-ping Hua

jinping_hua@cau.edu.cn

1 Laboratory of Cotton Genetics; Genomics and Breeding/Key Laboratory of Crop Heterosis and Utilization of Ministry of Education/Beijing Key Laboratory of Crop Genetic Improvement, College of Agronomy and Biotechnology, China Agricultural University, No. 2, Yuanmingyuan West Rd, Haidian district, Beijing 100193, China

2 Oil Crops Research Institute, Chinese Academy of Agricultural Sciences, Wuhan 430062, Hubei, China

3 Institute of Cash Crops, Hubei Academy of Agricultural Sciences, Wuhan 430064, Hubei, China
(QTL) for subsequent marker-assisted selection (MAS); (ii) generation of transgenic plants to affect the degree of salt stress tolerance (Yamaguchi and Blumwald 2005). Salt stress is known to repress plant growth due to osmotic stress, which is then followed by ion toxicity. Drastic changes in ion and water homeostasis lead to molecular damage, growth arrest, and even death (Flowers 2004; Zhu 2001; Wang and Huang 2019; Richter et al. 2019). To overcome salt stress, complicated adjustment to metabolic networks with multipronged responses involves fast-acting, immediate physiological responses, and long-term reactions has been highlighted in plants under high salinity conditions (Guo et al. 2015; Zhang et al. 2011; Liu et al. 2019). Complex multi-component signaling pathways in plants can be triggered face salinity stress, such as plant hormones, plant transcription factor families (Su et al. 2017a), lipids (Gao et al. 2019), aquaporins (Wang et al. 2019; Pawłowicz and Masajada 2019), CIPK(Ma et al. 2019a, b), Snf (sucrose non-fermenting)-1-related protein kinases (SnRK2) (Su et al. 2017b; Shinozawa et al. 2019), and mitogen-activated protein kinase (MAPK), ROS signaling (Ismail and Horie 2017; Wang and Huang 2019) and stomatal signaling (Golldack et al. 2014).

Previous studies investigating salt tolerance was performed in QTL mapping at the germination stage or during 
vegetative growth, such as in cotton (Diouf et al. 2017; Sun et al. 2018, 2019), rice (Kumar et al. 2015), barley (Mano and Takeda 1997), tomato (Foolad and Chen 1999), and soybean (Lee et al. 2004). A major QTL for salt tolerance in soybean was discovered in $106 \mathrm{~F}_{2}$-derived lines from the cross of 'S-100' (salt tolerant line) $\times$ 'Tokyo' (salt sensitive line) with $41 \%$ of total genetic variation for salt tolerance in the field (Lee et al. 2004). The gene (HKT1;4-A2) was identified responsible to salt tolerance based on the QTL Naxl, which was mapped as a salt stress related QTL and linked to the microsatellite marker gwm 312 on chromosome $2 \mathrm{AL}$ in durum wheat (Triticum monococcum) in durum wheat (Triticum monococcum) (Huang et al. 2006; Tounsi et al. 2016). Therefore, more researches involving QTL exploration salt tolerance are imperative for further development in crop.

The damaging effects of salt accumulation in agricultural soils affect crop productivity due to soil salinity. Upland cotton, as an important crop for renewable natural fiber source of textiles, is a pioneer crop in saline areas, because it is tolerant with salinity threshold of $7.7 \mathrm{dS} / \mathrm{m}(77 \mathrm{mM} \mathrm{NaCl})$, higher than soil saline with $4 \mathrm{dS} / \mathrm{m}(40 \mathrm{mM} \mathrm{NaCl})$ (Chinnusamy et al. 2006; Shi et al. 2015). The release of genome data of upland cotton has greatly facilitated cotton research (Zhang et al. 2015; Hu et al. 2019a, b; Chen et al. 2020). Cultivated upland cotton adapted to various environments and experienced periodic salinity extremes (Wendel et al. 2010), originating from $D$ sub-genome of response to abiotic stresses (Zhang et al. 2010). Eight SSR (simple sequence repeats) sites significantly associated with salt tolerance were found at the seedling stage through an association analysis in 134 cotton cultivars (Zhao et al. 2016). Comparative transcriptome analysis revealed that gas signaling process and ROS responding process enhanced abiotic stress in domesticated cotton and prolonged the duration of fiber elongation (Chaudhary et al. 2008, 2009; Park et al. 2012). One hundred and twenty-eight of the early differentially expressed proteins (DEPs) were identified from salt-treated cotton roots, 76 of which displayed increased abundance and 52 decreased under salt stress conditions in upland cotton (Li et al. 2015).

Obviously more exploration is needed in cotton, since no QTL under salt stress condition is included in 551 cotton QTL identified in Cottongen database resource (https:// www.cottongen.org) (Ijaz et al. 2019). Some QTL clusters and hotspots containing 661 QTL were collected for traits response to salt stress in cotton, of which, 80 QTL were detected for salt tolerance just in greenhouse condition but no QTL in field condition (Abdelraheem et al. 2017). A total of 11 consistent QTL were detected for seven traits in seedling stage in an $\mathrm{F}_{2: 3}$ population at $150 \mathrm{mM} \mathrm{NaCl}$ in a hydroponic environment (Oluoch et al. 2016). Recently, nine candidate intron length polymorphisms (ILPs) markers were verified using association mapping in a set of natural upland cotton accessions for salt stress in greenhouse condition (Cai et al. 2017). And the $C 4$ gene encoding WRKY DNA-binding protein and the $C 9$ gene encoding mitogenactivated protein kinase can significantly enhance cotton susceptibility to salt stress. The assessment of stress tolerance in the greenhouse often has little correlation with tolerance in the field (Yamaguchi and Blumwald 2005).

Cotton fiber is one of the most prevalent natural materials used in textile production. Fiber development consists of four stages (initiation, elongation, secondary cell wall biosynthesis, and maturation), which are defined and based on the number of days post-anthesis (DPA). For fiber development, the most active stage is the rapid elongation stage following the initiation stage and lasts up to 20 DPA (Kim et al. 2001). Studies have shown that the biosynthesis of very-long-chain fatty acids (VLCFAs, fatty acids $>$ C18) plays important roles in fiber development as well as the precursors of sphingolipids, seed triacylglycerols, suberin, and cuticular waxes (Qin et al. 2007; Qin and Zhu 2011; Hu et al. 2019a, b). VLCFAs may activate ethylene synthesis in cotton fiber elongation (Shi et al. 2006). KCS (3-ketoacyl-CoA synthase) is the first limiting enzyme in the biosynthesis steps of VLCFA, which determines the substrate and tissue specificities of the reaction in plants (Qin et al. 2007). It is reported that $21 \mathrm{KCS}$ genes were identified in the Arabidopsis genome with distinct tissue-specific, temporal-specific or spatial-specific expression patterns, reflecting their multiple roles in plant growth and development (Qin and Zhu 2011).

There is a lack of experimentation under natural salt stress conditions in the current literature. In the study, we evaluated field performance of five fiber quality traits under two conditions using a recombinant inbred lines (RIL) population derived from a cross of 'Xinza 1', an $\mathrm{F}_{1}$ hybrid upland cotton cultivar upland cotton. We performed QTL mapping of fiber quality traits under salt stress and normal growth conditions in three years in order to explore genetic basis of fiber development under salt stress in upland cotton. The findings of this research identify candidate genes associated with fiber elongation underlying the QTL and provide valuable insights for the improvement of cotton fiber quality.

\section{Materials and methods}

\section{Plant materials}

The RIL population was derived from a $\mathrm{F}_{1}$ hybrid 'Xinza 1' (GX1135 $\times$ GX100-2) by single seed descent method in upland cotton (Shang et al. 2015, 2016a), including 177 lines of $F_{15}-F_{17}$ generations. The control set was performed in two field trials, including GX1135, 'Xinza 1' $\mathrm{F}_{1}$, GX100-2, and a commercial hybrid 'Ruiza $816^{\prime}$ ' used as a competitive check (Ma et al. 2017, 2019a, b). 


\section{Field arrangement}

Two field trials under salt stress and normal conditions were conducted in 2016, 2017, and 2018 at Quzhou Experimental Station of China Agricultural University, Handan City, Hebei Province $\left(36^{\circ} 78^{\prime} \mathrm{N}, 114^{\circ} 92^{\prime} \mathrm{E}\right)$. Quzhou County is well known for the achievements in saline-alkali soil improvement since 1970's.

Two independent field trials were arranged in neighboring fields following a randomized complete block design with two replications each in 2016, 2017 and 2018. A total of 362 plots with two rows (22 individual plants per row) were conducted, respectively. Two repeats of 177 RI Lines $\left(F_{15}-F_{17}\right)$ were planted together with two control sets $\left(\mathrm{GX} 1135, \mathrm{~F}_{1}\right.$ 'Xinza 1', GX100-2, 'Ruiza 816'). Each plot was spaced $80 \mathrm{~cm}$ apart, and rows within plots spaced $60 \mathrm{~cm}$ apart. Plot lengths were $2.4 \mathrm{~m}$ in 2016, and $3.0 \mathrm{~m}$ in 2017 and 2018. A $0.7 \mathrm{~m}$ pavement separated the field experiments.

For salt stress treatment, shallow saline groundwater with concentration of $5 \mathrm{~g} / \mathrm{L}(85 \mathrm{mM})$ saline was used to irrigate the field twice in January and March before sowing. For control treatment, the regular irrigation with non-saline water was performed needed. Field management followed the local standard field practices.

\section{Soil sample collection and component detection}

Soil samples were collected from the $0-20 \mathrm{~cm}$ and $20-40 \mathrm{~cm}$ depth after sowing or before harvest. To cover the experiment area, sampling points were chosen every $15 \mathrm{~m}$ from north to south in the experiment field. Three soil samples collected for each sample site were mixed into one sample for soil quality determination. Soil saturated paste extracts (1:2 by weight) were prepared to measure the electric conductivity (EC) and total content of water-soluble salt $(\rho)$ (Rhoades 1996).

The measured sample properties of salinity from EC, and $\rho$ for each sampling points are summarized in Table S1.

\section{Fiber sample preparation and evaluation}

Twenty-five naturally opened bolls in the middle of plants were hand-harvested for each plot at crop maturity. A total of 367 and 363 fiber samples were prepared in 2016 on salt stress and normal conditions, respectively. In 2017, 362 and 358 fiber samples were collected from the two conditions. In 2018, a total of 362 fiber samples in each condition were collected. All samples were tested for five fiber quality traits with HVI 900 instrument (USTER_HVISPECTRUM, SPINLAB, USA) at Cotton Fiber Quality Inspection and Test Center of Ministry of Agriculture (Anyang, China). The fiber quality traits obtained were as follows: $2.5 \%$ fiber span length (for short fiber length, unit: $\mathrm{mm}$ ), fiber uniformity (\%), fiber strength (cN/tex), fiber elongation (\%), and fiber Micronaire (Shang et al. 2015; Ma et al. 2017).

\section{Dataset constitution and data conversion}

Three datasets of (1) salt stress condition (E1), (2) normal condition (E2), and (3) the difference values between salt stress and normal conditions ( $D$-value) were used in the present study. The original data of five fiber quality traits were obtained from the trials under E1 and E2, respectively. To ensure the $D$-value were positive, a constant $(\mathrm{C}=10)$ was added to convert the data prior to statistical analysis.

\section{DNA extraction and marker detection}

Genomic DNA was extracted from the parents and RIL plants using CTAB (Cetyltrimethylammonium bromide) method (Paterson et al. 1993). Genomic DNA of the RILs and two parents (GX1135 and GX100-2) was used to construct Illumina libraries with an insert size of 300-400 bp on the Illumina HiSeq platform. The clean reads were aligned to the G. hirsutum accession Texas Marker-1 (TM-1) reference genome using BWA software. The alignment files were converted into BAM files and then sorted using Samtools software (Li et al. 2009). The sorted reads in BAM files used in variant calling. SNP calling on a population scale was performed with the Genome Analysis Toolkit (GATK) (McKenna et al. 2010).

\section{Genetic linkage map construction}

Linkage map analysis was conducted using Join Map 4.0. The adjacent markers from the same parent were recorded as one bin (Xie et al. 2010). The linkage map was constructed after the repetition was removed from the markers within the distance of $10 \mathrm{~kb} .27,387 \mathrm{SNP}$ (single nucleotide polymorphism), or InDel (insertion or deletion) markers were divided into 26 linkage groups by Logarithm of Odds (LOD) $>9$, and 654 SSR markers involved in the original SSR genetic map (Shang et al. 2016b) were selected by LOD $>3$. Finally, a total of 330 SSR markers were distributed in the new linkage map. Recombination frequencies were converted into map distances (cM) using the Kosambi mapping function (Kosambi 1944).

The Chi-square test was to determine if the observed genetic segregation ratios of alleles were consistent with expected segregation ratios. A region on the genetic map with at least six adjacent loci showing significant segregation distortion $(P<0.05)$ was defined as the segregation distortion region (SDR). 


\section{Data analysis and QTL mapping}

The experimental data were analyzed by the software SPSS (Version 20.0, SPSS, Chicago). QTL mapping and the genetic effect values at single-locus level were conducted by QTL Cartographer software (Version 2.5) using the composite interval mapping (CIM) method (Zeng 1994; Wang et al. 2007). We set parameter in the confidence interval of $95 \%$ with composite interval mapping (CIM) method for QTL mapping. The threshold of LOD values were estimated after 1000 permutations tests to declare a significant QTL with a significance level of $P<0.05$, whereas QTL in another trial with LOD of at least 2.0 was considered as common QTL (Liang et al. 2013; Shang et al. 2015, 2016b). Common QTL were declared according to the position linked and if they shared one or two common markers (Shao et al. 2014).

\section{Candidate gene identification and annotation}

The genes located in the confidence intervals of the important QTL were fetched from the Cottongen (https://www. cottongen.org) using their flanking marker positions in $G$. hirsutum TM-1 genome (Zhang et al. 2015) and considered as candidate genes. Gene ontology (GO) enrichment and KEGG pathway analysis were carried out for all candidate genes. The GO enrichment was performed using GO databases (https://archive.geneontology.org/latest-lite/). To further screen the possible candidate genes involved in fiber development, the gene expression pattern of candidate genes in different period of fiber development was analyzed using the cotton functional genome database (https://www.cotto nfgd.org).

\section{RNA extraction and gene expression validation}

Total RNA was isolated from $0,5,10,15$, and 20 DPA fiber in extremely long fiber length line $(\mathrm{H})$, extremely short fiber length line (L), female (GX1135, F), and male (GX100-2, $\mathrm{M})$. The concentration and purity of total RNA were determined using the Nano Drop spectrophotometry and agarose gel electrophoresis, respectively. RNA samples were stored at $-80{ }^{\circ} \mathrm{C}$ freezer for future use.

To validate the potential function in fiber development, the expression patterns of candidate genes were verified with qRT-PCR using RNA of fiber in different development period of extremely lines in fiber length trait and parents of the population. Gene relative expression level was calculated with $2^{-\Delta \Delta C t}$ method (Livak and Schmittgen 2001). Primers for the qRT-PCR analysis are listed in Table S3. Three independent replicates were performed for each sample. $G h U B Q 7$ gene was used as a reference gene.

\section{Results}

\section{Phenotypic performance of fiber quality traits under salt stress and normal conditions}

According to the grading standard of saline soil, the average EC of 0-20 cm soil samples collected during three years trial showed moderate salinization (323.11-648.22 $\left.\mu \mathrm{s} \cdot \mathrm{cm}^{-1}\right)$ in saline soil and mild salinization (350.08-455.33 $\left.\mu \mathrm{s} \mathrm{cm}^{-1}\right)$ in normal soil, respectively (Table S1, Table S2) (Wang et al. 1993). The average EC of $20-40 \mathrm{~cm}$ was moderate salinization $\left(656.78-943.92 \mu \mathrm{s} \mathrm{cm}^{-1}\right)$ in saline soil and mild salinization (299.22-427.42 $\mu \mathrm{s} \mathrm{cm}^{-1}$ ), respectively. It is indicated that there was a significant difference in salt concentration between the two areas after saline irrigation (Wang et al. 1993; Table S2).

For the datasets of E1, E2, and $D$-value, phenotypic performance of fiber quality traits is shown in Tables 1 and 2, including all lines from the RIL population, $\mathrm{F}_{1}$ hybrid 'Xinza 1 ', female parent GX1135, male parent GX100-2, and competitive check hybrid 'Ruiza 816' (Shang et al. 2015).

The performance of maternal parent GX1135 was often superior to that of male parent GX100-2 whether on E1 or E2. For GX1135 and GX100-2 with homozygous alleles, the trend of phenotypic values on E1 and E2 for fiber length (FL), fiber uniformity (FU) ratio and fiber strength (FS) across three years was not consistent. The phenotypic values on salt stress condition increased much for Micronaire (FM), but had no significant change for fiber elongation (FE) (Table 1). Moreover, mean value of $F_{1}$ hybrid 'Xinza 1' for FM showed no incremental change. It revealed that responding mechanisms to salt stress may differ in the hybrid and its parents (Table 1).

In the RIL population, skewness and kurtosis values of five fiber quality traits ranged between -1.00 and 1.00 for both growing conditions, except that of FU under E1 in 2016 and 2017 (Table 1). Five fiber quality traits showed an abundance of variation in the in the RIL population either on E1 or E2. All fiber quality traits exhibited coefficient of variation less than $15 \%$, indicating that the original data for these traits was reliable. Compared with E2, phenotypic values in the RIL population decreased for FL, FU and FS, but increased for FM and FE under E1 in 2016. In 2017, phenotypic values under E1 were increased for FL, FU, FS, and FE, but decreased for FM. In 2018, phenotypic values under E1 were decreased for FL, FU, and FE, however, increased for FS. These results indicate that the data obtained were credible. 
Table 1 Descriptive statistical analysis for fiber quality traits under salt stress and normal conditions

\begin{tabular}{|c|c|c|c|c|c|c|c|c|c|c|c|c|c|}
\hline \multirow[t]{2}{*}{ Traits } & \multirow[t]{2}{*}{ Year } & \multirow[t]{2}{*}{ Environment } & \multicolumn{7}{|c|}{ Populations } & \multicolumn{2}{|l|}{ Parents } & \multirow{2}{*}{$\begin{array}{l}F_{1} \\
\text { Xinza1 }\end{array}$} & \multirow{2}{*}{$\begin{array}{l}\text { CK } \\
\text { Ruiza } 816\end{array}$} \\
\hline & & & Mean & $\mathrm{SD}^{\mathrm{b}}$ & $\mathrm{CV} \%^{\mathrm{c}}$ & Min & Max & Skewness & Kurtosis & GX1135 & GX100-2 & & \\
\hline \multirow[t]{6}{*}{$\mathrm{FL}(\mathrm{mm})^{\mathrm{a}}$} & 2016 & E1 & 30.28 & 1.17 & 3.85 & 26.75 & 33.90 & -0.13 & 0.31 & 30.23 & 29.70 & 30.43 & 31.00 \\
\hline & 2016 & E2 & 30.30 & 1.15 & 3.80 & 27.45 & 34.20 & 0.07 & 0.28 & 30.43 & 29.90 & 30.83 & 31.05 \\
\hline & 2017 & E1 & 30.52 & 1.15 & 3.78 & 26.70 & 33.05 & -0.28 & 0.05 & 30.30 & 30.95 & 30.40 & 29.35 \\
\hline & 2017 & E2 & 30.07 & 1.07 & 3.57 & 27.15 & 33.50 & 0.21 & 0.06 & 29.00 & 29.70 & 29.30 & 29.60 \\
\hline & 2018 & E1 & 29.39 & 1.08 & 3.66 & 26.50 & 32.10 & 0.08 & -0.27 & 28.05 & 28.70 & 28.45 & 30.25 \\
\hline & 2018 & E2 & 29.79 & 1.14 & 3.82 & 26.25 & 32.60 & -0.05 & -0.30 & 29.05 & 29.20 & 30.50 & 29.95 \\
\hline \multirow[t]{6}{*}{ FU (\%) } & 2016 & E1 & 85.53 & 0.85 & 1.00 & 82.15 & 87.45 & -0.62 & 1.36 & 85.63 & 85.30 & 85.35 & 85.25 \\
\hline & 2016 & E2 & 85.77 & 0.73 & 0.85 & 83.90 & 87.40 & -0.31 & -0.22 & 86.30 & 85.55 & 86.53 & 85.20 \\
\hline & 2017 & E1 & 85.55 & 0.76 & 0.89 & 81.80 & 87.30 & -0.82 & 2.90 & 84.80 & 86.00 & 85.85 & 86.40 \\
\hline & 2017 & E2 & 84.76 & 0.86 & 1.01 & 82.90 & 87.10 & 0.01 & -0.57 & 85.30 & 85.30 & 86.35 & 86.65 \\
\hline & 2018 & E1 & 83.15 & 1.10 & 1.32 & 80.05 & 85.70 & -0.22 & -0.39 & 81.40 & 84.40 & 82.65 & 85.10 \\
\hline & 2018 & E2 & 84.36 & 0.89 & 1.06 & 82.10 & 86.80 & -0.12 & 0.01 & 82.55 & 84.25 & 85.45 & 85.35 \\
\hline \multirow[t]{6}{*}{ FS (cN/tex) } & 2016 & E1 & 28.58 & 1.22 & 4.25 & 25.80 & 31.65 & -0.07 & -0.24 & 30.45 & 27.60 & 28.60 & 30.43 \\
\hline & 2016 & E2 & 29.71 & 1.23 & 4.15 & 26.35 & 33.35 & -0.09 & 0.14 & 30.43 & 29.45 & 29.95 & 31.15 \\
\hline & 2017 & E1 & 28.98 & 1.64 & 5.68 & 25.45 & 34.40 & 0.22 & 0.16 & 28.95 & 29.00 & 29.00 & 29.75 \\
\hline & 2017 & E2 & 28.08 & 1.48 & 5.27 & 24.65 & 32.40 & 0.17 & -0.07 & 30.50 & 26.40 & 27.45 & 30.60 \\
\hline & 2018 & E1 & 31.49 & 1.83 & 5.80 & 26.55 & 36.30 & 0.11 & -0.02 & 31.35 & 30.10 & 29.95 & 33.90 \\
\hline & 2018 & E2 & 30.46 & 1.75 & 5.75 & 26.05 & 34.90 & 0.07 & -0.31 & 30.85 & 28.65 & 32.40 & 31.75 \\
\hline \multirow[t]{6}{*}{$\mathrm{FE}(\%)$} & 2016 & E1 & 6.81 & 0.07 & 1.06 & 6.65 & 7.05 & 0.01 & 0.43 & 6.80 & 6.75 & 6.83 & 6.80 \\
\hline & 2016 & E2 & 6.78 & 0.08 & 1.17 & 6.50 & 6.95 & -0.23 & 0.37 & 6.78 & 6.78 & 6.88 & 6.83 \\
\hline & 2017 & E1 & 6.87 & 0.09 & 1.25 & 6.60 & 7.10 & -0.12 & 0.27 & 6.75 & 6.75 & 6.85 & 6.75 \\
\hline & 2017 & E2 & 6.80 & 0.07 & 1.10 & 6.60 & 7.05 & 0.13 & 0.46 & 6.75 & 6.75 & 6.85 & 6.75 \\
\hline & 2018 & E1 & 5.61 & 0.30 & 5.42 & 4.75 & 6.70 & 0.32 & 0.50 & 5.85 & 5.60 & 5.75 & 5.80 \\
\hline & 2018 & E2 & 5.70 & 0.33 & 5.72 & 4.65 & 6.45 & -0.33 & 0.17 & 5.75 & 5.50 & 6.00 & 5.80 \\
\hline \multirow[t]{6}{*}{ FM } & 2016 & E1 & 4.95 & 0.39 & 7.93 & 3.90 & 5.85 & -0.03 & -0.48 & 4.98 & 4.95 & 4.85 & 5.03 \\
\hline & 2016 & E2 & 4.83 & 0.38 & 7.93 & 4.05 & 5.75 & 0.23 & -0.73 & 4.78 & 4.40 & 5.10 & 5.10 \\
\hline & 2017 & E1 & 5.53 & 0.31 & 5.62 & 4.70 & 6.35 & -0.22 & -0.08 & 5.95 & 5.60 & 5.65 & 5.85 \\
\hline & 2017 & E2 & 5.58 & 0.28 & 5.02 & 4.60 & 6.25 & -0.38 & 0.57 & 5.70 & 5.55 & 5.95 & 5.75 \\
\hline & 2018 & E1 & 6.80 & 0.04 & 0.66 & 6.65 & 6.90 & -0.29 & 0.88 & 6.75 & 6.80 & 6.70 & 6.80 \\
\hline & 2018 & E2 & 6.80 & 0.05 & 0.80 & 6.70 & 6.95 & -0.01 & -0.13 & 6.75 & 6.80 & 6.90 & 6.75 \\
\hline
\end{tabular}

E1, salt stress condition; E2, normal condition

${ }^{\mathrm{a}} \mathrm{FL}$, fiber length/mm, FU, fiber uniformity ratio/\%, FS, fiber strength(cN/tex), FE, fiber elongation/\%, FM, Micronaire. Here in after same

${ }^{\mathrm{b}}$ Mean values \pm standard deviation values

${ }^{\mathrm{c} C o e f f i c i e n t}$ of variation

\section{Correlation analysis between fiber quality traits}

Table 3 presents correlation analysis on five fiber quality traits using datasets of mean phenotypic values on E1, E2, and $D$-value.

Under E2, FL showed significant positive correlation with FS, FE, and FU, but was negatively correlated with FM during the three years of this trial. FS showed significant positive correlation with FE, and negative correlation with FM in three years. No significant correlation was observed between FU and FM. The results under E2 showed similar tendency and compared similarly to previous results (Liang et al. 2013; Shang et al. 2015). It revealed that the original data were reasonable for dissecting the genetic basis of fiber quality traits under both growing conditions.

Under E1, FL showed significant positive correlation with FS and FE, but showed negative correlation with FM in 2016, and significant positive correlation with FM in 2017. FU showed significant positively correlation with FE and FM during three years. FS showed significant positively correlation with FE each year, and significant positive correlated with FM in 2017, but was negatively with FM in 2016 and in 2018. 
Table 2 Descriptive statistical analysis for fiber quality traits on the difference value dataset between salt stress condition and normal condition

\begin{tabular}{|c|c|c|c|c|c|c|c|c|c|c|c|c|}
\hline Traits & Year & Mean & SD & CV\% & Min & Max & Skewness & Kurtosis & GX1135 & GX100-2 & Xinza1 & Ruiza 816 \\
\hline \multirow[t]{3}{*}{$\mathrm{FL}(\mathrm{mm})$} & 2016 & 9.99 & 0.95 & 9.53 & 4.95 & 12.70 & -0.57 & 4.26 & 9.80 & 9.80 & 9.60 & 9.95 \\
\hline & 2017 & 10.44 & 0.98 & 9.39 & 7.85 & 12.90 & -0.10 & -0.41 & 11.30 & 11.25 & 11.10 & 9.75 \\
\hline & 2018 & 9.60 & 0.84 & 8.79 & 7.55 & 12.35 & 0.00 & 0.02 & 9.00 & 9.50 & 7.95 & 10.30 \\
\hline \multirow[t]{3}{*}{ FU (\%) } & 2016 & 9.76 & 0.97 & 9.97 & 5.40 & 12.70 & -0.60 & 2.54 & 9.33 & 9.75 & 8.82 & 10.05 \\
\hline & 2017 & 10.79 & 1.00 & 9.23 & 7.45 & 13.20 & -0.28 & 0.23 & 9.50 & 10.70 & 9.50 & 9.75 \\
\hline & 2018 & 8.78 & 1.29 & 14.69 & 5.55 & 12.20 & 0.09 & -0.18 & 8.85 & 10.15 & 7.20 & 9.75 \\
\hline \multirow[t]{3}{*}{$\mathrm{FS}(\mathrm{cN} / \mathrm{tex})$} & 2016 & 8.87 & 1.21 & 13.64 & 5.00 & 12.65 & 0.03 & 0.96 & 10.02 & 8.15 & 8.65 & 9.28 \\
\hline & 2017 & 10.90 & 1.52 & 13.96 & 6.65 & 15.70 & 0.14 & -0.11 & 8.45 & 12.60 & 11.55 & 9.15 \\
\hline & 2018 & 11.01 & 1.66 & 15.07 & 6.00 & 15.30 & -0.34 & -0.03 & 10.50 & 11.45 & 7.55 & 12.15 \\
\hline \multirow[t]{3}{*}{$\mathrm{FE}(\%)$} & 2016 & 10.02 & 0.08 & 0.82 & 9.70 & 10.35 & 0.37 & 2.34 & 10.02 & 9.97 & 9.95 & 9.97 \\
\hline & 2017 & 10.07 & 0.08 & 0.84 & 9.85 & 10.35 & 0.11 & 0.14 & 10.00 & 10.00 & 10.00 & 10.00 \\
\hline & 2018 & 10.00 & 0.05 & 0.55 & 9.80 & 10.15 & -0.50 & 1.22 & 10.10 & 10.10 & 9.75 & 10.00 \\
\hline \multirow[t]{3}{*}{ FM } & 2016 & 10.12 & 0.40 & 3.97 & 8.50 & 11.15 & -0.19 & 1.09 & 10.20 & 10.55 & 9.75 & 9.93 \\
\hline & 2017 & 9.96 & 0.19 & 1.95 & 9.20 & 10.70 & -0.15 & 2.45 & 10.25 & 10.05 & 9.70 & 10.10 \\
\hline & 2018 & 9.91 & 0.20 & 2.00 & 9.45 & 10.50 & 0.30 & 0.11 & 10.00 & 10.00 & 9.80 & 10.05 \\
\hline
\end{tabular}

For FM, significant negative correlation existed with FL and FS under E2, but the correlative tendency under E1 was not consistent each year. FU showed significant positive correlation with FL and FS under E2 and showed significant positive correlation or no significant correlation under E1. It revealed that salt stress reduces the correlation between FL and FU, as well as FS and FU. FU showed significant positive correlation with FE and FM over three years, while the correlation between FU and FE, FU, and FM under E2 showed decreased, it indicated that salt stress enhanced the correlation dramatically. Correlations between FE and FM were not significant under E1 and E2. FE showed positive significant correlation with FS under both conditions. It indicated that salt stress had no effect on the correlations between FE and FS, FE, and FM.

\section{Construction of genetic map}

Among the 174,351 markers, the low-coverage sequences of the RIL populations were filtered out, leaving 34,361 markers. After filtering SNPs according to the genotyping criteria, a total of 27,387 homozygous markers were identified between the two parents to generate bin markers for the RIL population. Finally, a total of 27,387 polymorphic SNP or Indel markers and 330 SSR markers were used for the construction of linkage map. Adjacent markers with the same genotype were merged as an identical bin. A highdensity genetic linkage map was constructed with 2859 recombination bin markers. Distribution of markers and marker interval on chromosomes based on RIL linkage map is shown in Table 4. The genetic map covered $2133.53 \mathrm{cM}$ of cotton genome with average interval of $0.785 \mathrm{cM}$. Among the 26 linkage groups, the average bin interval was
$5.979 \mathrm{cM}$, and the range of interval on 26 chromosomes was 3.647-10.361 cM, and only one gap larger than $10 \mathrm{cM}$ was observed in chromosome D05. The length of the linkage groups ranged from $34.181 \mathrm{cM}$ (A09) to $149.966 \mathrm{cM}$ (A05). The linkage map harboring these markers is shown in Table S4 and Fig. S1. On an average, one linkage group harbored about 110 bins that covered an average of $82.059 \mathrm{cM}$. The average bin interval was $0.758 \mathrm{cM}$ with a range of $0.523 \mathrm{cM}$ (A13) to $0.939 \mathrm{cM}$ (D01). The highest number of markers (181) were present in the chromosome A05 with an average marker interval of $1.207 \mathrm{cM}$.

\section{Segregation distortion}

Among the 2859 polymorphic loci, 336 (11.75\%) showed segregation distortion $(P<0.05)$ with $213(63.39 \%)$ favoring the GX1135 alleles and 123 (36.61\%) favoring the GX100-2 alleles (Table S5). These distorted loci existed on 19 chromosomes and mapped unevenly on different chromosomes. More distorted loci were located on Dt subgenome than on At subgenome (142 versus 194). Among them, Chr. D01 and D04 accounted for $61.36 \%$ and $51.32 \%$ of distorted loci on corresponding chromosome, respectively (Table S5). There are $280(83.33 \%)$ of the 336 distorted loci were clustered into 15 SDRs, with nine located on At subgenome and six on Dt subgenome. Chr. D11 was heavily concentrated with distorted loci, with 51 loci showing significant distortion toward GX100-2, forming a large SDR of $40.451 \mathrm{cM}$ (Table S6). SDR 12 which located on Chr. D04 showing significant distortion toward GX1135 and forming the largest interval of $40.561 \mathrm{cM}$ (Table S6). 
Table 3 Correlation analyses for five fiber quality traits under salt stress condition, normal condition, and by phenotypic differences

\begin{tabular}{|c|c|c|c|c|c|c|}
\hline Environment & Trait & Year & FL & FU & FS & $\mathrm{FE}$ \\
\hline \multirow[t]{12}{*}{ E1 } & FU & 2016 & -0.093 & & & \\
\hline & FS & 2016 & $0.693 * *$ & -0.132 & & \\
\hline & FE & 2016 & $0.560 * *$ & $0.164^{*}$ & $0.510 * *$ & \\
\hline & FM & 2016 & $-0.519 * *$ & $0.349 * *$ & $-0.489 * *$ & -0.143 \\
\hline & FU & 2017 & $0.294 * *$ & & & \\
\hline & FS & 2017 & $0.768 * *$ & $0.321 * *$ & & \\
\hline & $\mathrm{FE}$ & 2017 & $0.559 * *$ & $0.773 * *$ & $0.578 * *$ & \\
\hline & FM & 2017 & $0.259 * *$ & $0.756 * *$ & $0.274 * *$ & $0.840 * *$ \\
\hline & FU & 2018 & $0.196^{* *}$ & & & \\
\hline & FS & 2018 & $0.452 * *$ & -0.017 & & \\
\hline & $\mathrm{FE}$ & 2018 & $0.579 * *$ & $0.212 * *$ & $0.540 * *$ & \\
\hline & FM & 2018 & -0.122 & $0.234 * *$ & $-0.453 * *$ & -0.011 \\
\hline \multirow[t]{12}{*}{ E2 } & FU & 2016 & $0.380^{* *}$ & & & \\
\hline & FS & 2016 & $0.541 * *$ & $0.273 * *$ & & \\
\hline & FE & 2016 & $0.372 * *$ & $0.212 * *$ & $0.331 * *$ & \\
\hline & FM & 2016 & $-0.390 * *$ & -0.026 & $-0.240 * *$ & 0.126 \\
\hline & FU & 2017 & $0.159^{*}$ & & & \\
\hline & FS & 2017 & $0.528 * *$ & $0.210 * *$ & & \\
\hline & $\mathrm{FE}$ & 2017 & $0.382 * *$ & 0.143 & $0.388 * *$ & \\
\hline & FM & 2017 & $-0.509 * *$ & $-0.194 * *$ & $-0.289 * *$ & -0.064 \\
\hline & FU & 2018 & $0.232 * *$ & & & \\
\hline & FS & 2018 & $0.415^{* *}$ & $0.178^{*}$ & & \\
\hline & $\mathrm{FE}$ & 2018 & $0.571 * *$ & $0.171^{*}$ & $0.587 * *$ & \\
\hline & FM & 2018 & $-0.169^{*}$ & 0.013 & $-0.168^{*}$ & 0.103 \\
\hline \multirow[t]{12}{*}{$D$ - value } & FU & 2016 & $0.379 * *$ & & & \\
\hline & FS & 2016 & $0.567 * *$ & $0.366 * *$ & & \\
\hline & $\mathrm{FE}$ & 2016 & $0.514 * *$ & $0.454 * *$ & $0.534 * *$ & \\
\hline & FM & 2016 & $-0.516^{* *}$ & 0.112 & $-0.165^{*}$ & -0.001 \\
\hline & FU & 2017 & $0.463 * *$ & & & \\
\hline & FS & 2017 & $0.544 * *$ & $0.378 * *$ & & \\
\hline & $\mathrm{FE}$ & 2017 & $0.524 * *$ & $0.271 * *$ & $0.588 * *$ & \\
\hline & FM & 2017 & $-0.503 * *$ & -0.091 & $-0.194 * *$ & 0.027 \\
\hline & FU & 2018 & $0.296 * *$ & & & \\
\hline & FS & 2018 & $0.381 * *$ & $0.178^{*}$ & & \\
\hline & FE & 2018 & $0.587 * *$ & 0.100 & $0.552 * *$ & \\
\hline & FM & 2018 & -0.098 & 0.090 & -0.126 & 0.053 \\
\hline
\end{tabular}

Critical value for correlation coefficients at probabilities of 0.05 and 0.01 are 0.145 and 0.190 , respectively. E1, salt stress condition; E2, normal condition; $D$-value, the difference values between salt stress and normal conditions

*There was a significant correlation at 0.05 level (bilateral)

*** There was a significant correlation at 0.01 level (bilateral)

\section{QTL mapping for fiber quality}

A total of 159 QTL controlling fiber quality traits were detected under E1 and E2, explaining 2.71-16.03\% of total phenotypic variance (PV) (Table 5). Separately, 51, 70, and 53 QTL were detected in the RIL population under E1, E2, and $D$-value. Twelve QTL were detected in two or three years, of which seven, one, four, one, and one QTL were detected for FL, FU, FS, FE, and FM, respectively. A total of 10 QTL were identified in at least two datasets, three QTL were detected in E1 and E2 for FL, and one QTL was detected on E1 and $D$-value for FS. For FM, six QTL were detected on two datasets: three QTL on E1 and E2, two QTL on E2 and $D$-value, one QTL on E1 and $D$-value. However, no QTL were detected for FE and FU. 
Table 4 Distribution of markers and marker interval on chromosomes based on RIL linkage map

\begin{tabular}{|c|c|c|c|c|c|c|}
\hline Chr & Length(cM) & No. of markers & No. of bins & $\begin{array}{l}\text { Marker } \\
\text { interval } \\
(\mathrm{cM})\end{array}$ & Bin interval (cM) & Max interval (cM) \\
\hline A01 & 72.378 & 1646 & 109 & 0.044 & 0.670 & 6.410 \\
\hline A02 & 63.522 & 1115 & 98 & 0.057 & 0.655 & 3.979 \\
\hline A03 & 107.311 & 810 & 142 & 0.133 & 0.761 & 4.311 \\
\hline A04 & 76.875 & 1199 & 138 & 0.064 & 0.561 & 6.410 \\
\hline A05 & 149.966 & 960 & 181 & 0.156 & 0.833 & 5.690 \\
\hline A06 & 65.590 & 3618 & 104 & 0.018 & 0.637 & 3.979 \\
\hline A07 & 106.389 & 1021 & 129 & 0.104 & 0.831 & 6.050 \\
\hline A08 & 72.144 & 476 & 87 & 0.152 & 0.839 & 4.648 \\
\hline A09 & 34.181 & 583 & 45 & 0.059 & 0.777 & 4.991 \\
\hline A10 & 83.374 & 1170 & 107 & 0.071 & 0.787 & 5.365 \\
\hline A11 & 89.789 & 1783 & 129 & 0.050 & 0.701 & 5.690 \\
\hline A12 & 64.627 & 1094 & 104 & 0.059 & 0.627 & 3.647 \\
\hline A13 & 54.408 & 2366 & 105 & 0.023 & 0.523 & 3.647 \\
\hline D01 & 81.679 & 588 & 88 & 0.139 & 0.939 & 7.153 \\
\hline D02 & 103.223 & 1266 & 145 & 0.082 & 0.717 & 7.154 \\
\hline D03 & 87.538 & 1191 & 115 & 0.074 & 0.768 & 3.647 \\
\hline D04 & 69.669 & 326 & 76 & 0.214 & 0.929 & 6.781 \\
\hline D05 & 84.896 & 636 & 92 & 0.134 & 0.933 & 10.361 \\
\hline D06 & 85.986 & 787 & 124 & 0.109 & 0.699 & 5.340 \\
\hline D07 & 117.105 & 1227 & 153 & 0.096 & 0.770 & 6.049 \\
\hline D08 & 89.787 & 1078 & 120 & 0.083 & 0.755 & 6.050 \\
\hline D09 & 78.327 & 398 & 88 & 0.197 & 0.900 & 8.306 \\
\hline D10 & 65.318 & 394 & 78 & 0.166 & 0.848 & 8.305 \\
\hline D11 & 94.903 & 507 & 113 & 0.188 & 0.847 & 8.305 \\
\hline D12 & 87.626 & 558 & 113 & 0.157 & 0.782 & 6.410 \\
\hline D13 & 46.917 & 590 & 76 & 0.080 & 0.626 & 6.781 \\
\hline Whole & 2133.528 & 27,387 & 2859 & 0.104 & 0.758 & 5.979 \\
\hline
\end{tabular}

For FL, a total of 40 QTL located on 19 chromosomes were identified. Of those, 14 QTL was detected under E1, 17 and 11 QTL were identified in RIL population under E1 and $D$-value, respectively (Table 5). Seven QTL detected on E1 increased FL with threshold of LOD 2.62 on average. Ten QTL decreased FL explained $6.53 \%$ of PV on average. The $q F L-C h r 1-1, q F L-C h r 5-5, q F L-C h r 24-4$, which were detected on both E1 and E2, explained 3.68, 11.65, and $4.09 \%$ of PV, respectively. Three QTL were mapped with LOD threshold $2.27,6.72$, and 3.87 , respectively. Two of these QTL were identified in at least two years.

Out of 26 QTL (LOD ranged from 2.04 to 4.29), 4, 13, and 9 QTL for FU was identified on E1, E2, and $D$-value, respectively. Four QTL were identified in more than two years, but no QTL were detected in more than two growing conditions in the same year.

For FS, a total of 28 QTL were resolved explaining from 3.66 to $12.90 \%$ of PV, among which seven, 12, and 13 QTL were identified on E1, E2, and $D$-value, respectively. One QTL ( $q F S-C h r 25-2)$ by the threshold of LOD 5.79 was detected on both E1 and $D$-value. Two of three QTL located on Chr 24 explained PV more than $10 \%$.

Nine, 15, and five QTL for FE distributed on 18 chromosomes were identified under E1, E2, and $D$-value, respectively. The stable QTL $q F E$-Chr20-3, identified in the $D$-value, was also identified on E2 in 2016 and 2017 , with the threshold of LOD 3.44 on average. The QTL, $q F E$-Chr7-1, was identified under E2 and $D$-value with the threshold of LOD 4.31 on average. Identified in both E1 and $D$-value, $q F E$-Chr15-1 exhibited average LOD of 2.31 .

A total of 12, 18, and 8 QTL underlying FM were identified on 16 chromosomes under E1, E2, and $D$-value, respectively. Of these QTL, qFM-Chr8-3, qFM-Chr15-1, qFMChr15-3, and $q F M-C h r 21-1$ were detected repeatedly across two or three years. Three QTL detected on E1 also played important roles in controlling FM on E2, including $q F M$ Chr5-2, qFM-Chr8-2, and qFM-Chr8-3. Another two QTL were detected on E2 and D-value; these were $q F M$-Chr15-1 and $q F M-C h r 16-1$. One QTL, $q F M-C h r 25-1$, explained $4.60 \%$ of PV and was identified under E1 and $D$-value. 
Table 5 Single locus QTL for fiber quality traits under normal condition, salt stress and mapping by the difference

\begin{tabular}{|c|c|c|c|c|c|c|c|c|c|c|c|c|c|}
\hline \multirow[t]{2}{*}{ Trait } & \multirow[t]{2}{*}{ QTL } & \multirow[t]{2}{*}{ Year } & \multicolumn{2}{|c|}{ Flanking markers } & \multicolumn{3}{|c|}{ Under $\mathrm{E} 1^{\mathrm{c}}$} & \multicolumn{3}{|c|}{ Under E2 } & \multicolumn{3}{|c|}{ Mapping by $D$-value } \\
\hline & & & & & LOD & Effect value & $\operatorname{Var} \%^{\mathrm{b}}$ & LOD & Effect value & $\operatorname{Var} \%$ & LOD & Effect value & $\operatorname{Var} \%$ \\
\hline \multirow[t]{38}{*}{ FL } & $q F L-C h r l-1 *^{\mathrm{a}}$ & 2016 & $\operatorname{Bin} 28$ & Bin29 & 2.01 & 0.22 & 3.31 & 2.27 & 0.23 & 3.68 & & & \\
\hline & & 2018 & $\operatorname{Bin} 48$ & Bin49 & & & & 2.90 & -0.28 & 5.83 & & & \\
\hline & & 2018 & Bin51 & $\operatorname{Bin} 52$ & 2.12 & -0.22 & 4.23 & & & & & & \\
\hline & $q F L-C h r 4-1$ & 2016 & Bin 400 & Bin401 & 2.44 & 0.24 & 4.20 & & & & & & \\
\hline & $q F L-C h r 4-2$ & 2016 & Bin421 & Bin 422 & 2.48 & 0.24 & 4.06 & & & & & & \\
\hline & $q F L-C h r 5-1$ & 2016 & Bin490 & Bin491 & & & & 4.61 & -0.34 & 8.20 & & & \\
\hline & & 2017 & Bin493 & Bin494 & 6.49 & -0.40 & 12.11 & & & & & & \\
\hline & $q F L-C h r 5-2$ & 2016 & Bin500 & Bin501 & 2.79 & -0.26 & 4.87 & & & & & & \\
\hline & & 2017 & Bin502 & Bin503 & 5.08 & -0.36 & 9.66 & & & & & & \\
\hline & $q F L-C h r 5-3$ & 2016 & Bin512 & Bin513 & & & & 5.87 & -0.37 & 10.31 & & & \\
\hline & & 2017 & Bin515 & Bin516 & & & & 6.23 & -0.36 & 11.06 & & & \\
\hline & $q F L-C h r 5-4$ & 2017 & Bin527 & Bin528 & & & & 6.90 & -0.38 & 12.14 & & & \\
\hline & $q F L-C h r 5-5 *$ & 2017 & Bin648 & Bin649 & & & & 3.29 & -0.26 & 5.68 & & & \\
\hline & & 2016 & Bin648 & Bin649 & 5.91 & -0.39 & 10.80 & 6.73 & -0.40 & 11.65 & & & \\
\hline & $q F L-C h r 6-1$ & 2016 & Bin760 & Bin761 & 3.84 & 0.31 & 6.82 & & & & & & \\
\hline & $q F L-C h r 7-1$ & 2016 & Bin795 & Bin796 & & & & & & & 2.49 & 0.23 & 5.57 \\
\hline & $q F L-C h r 7-2$ & 2016 & Bin837 & Bin838 & & & & & & & 2.30 & -0.22 & 4.85 \\
\hline & $q F L-C h r 7-3$ & 2017 & Bin844 & Bin845 & & & & 3.34 & -0.28 & 6.35 & & & \\
\hline & $q F L-C h r 7-4$ & 2017 & Bin851 & Bin852 & & & & 2.30 & -0.22 & 4.02 & & & \\
\hline & $q F L-C h r 9-1$ & 2018 & Bin1024 & Bin 1025 & 2.35 & -0.24 & 4.70 & & & & & & \\
\hline & $q F L-C h r 11-1$ & 2017 & Bin1168 & Bin1169 & & & & & & & 2.23 & -0.21 & 4.48 \\
\hline & $q F L-C h r 12-1$ & 2018 & Bin1368 & Bin1369 & & & & & & & 2.97 & -0.22 & 6.17 \\
\hline & & 2018 & Bin1371 & Bin 1372 & & & & 2.72 & 0.27 & 5.45 & & & \\
\hline & $q F L-C h r 13-1$ & 2017 & Bin1381 & Bin1382 & 3.67 & -0.30 & 6.60 & & & & & & \\
\hline & $q F L-C h r 13-2$ & 2017 & Bin1389 & Bin1390 & 3.26 & -0.29 & 6.14 & & & & & & \\
\hline & $q F L-C h r 14-1$ & 2018 & Bin1483 & Bin1484 & 2.33 & -0.24 & 4.64 & & & & & & \\
\hline & $q F L-C h r 14-2$ & 2018 & Bin 1517 & Bin 1518 & & & & 2.13 & -0.23 & 4.01 & & & \\
\hline & $q F L-C h r 14-3$ & 2016 & Bin1529 & Bin 1530 & & & & & & & 2.13 & 0.21 & 4.40 \\
\hline & $q F L-C h r 15-1$ & 2017 & Bin1599 & Bin 1600 & & & & & & & 2.47 & -0.23 & 5.15 \\
\hline & $q F L-C h r 15-2$ & 2017 & Bin1694 & Bin1695 & & & & 2.31 & 0.22 & 3.71 & & & \\
\hline & & 2018 & Bin1707 & Bin1708 & & & & 2.02 & 0.23 & 4.00 & & & \\
\hline & $q F L-C h r 16-1$ & 2016 & Bin1760 & Bin1761 & & & & 2.13 & -0.21 & 3.26 & & & \\
\hline & $q F L-C h r 16-2$ & 2017 & Bin1784 & Bin1785 & & & & 2.10 & -0.20 & 3.38 & & & \\
\hline & $q F L-C h r 18-1$ & 2017 & Bin1933 & Bin1934 & & & & 2.07 & -0.21 & 3.33 & & & \\
\hline & $q F L-C h r 19-1$ & 2017 & Bin1996 & Bin1997 & 2.38 & -0.24 & 4.21 & & & & & & \\
\hline & $q F L-C h r 21-1$ & 2017 & $\operatorname{Bin} 2335$ & $\operatorname{Bin} 2336$ & & & & 2.84 & 0.24 & 4.87 & & & \\
\hline & $q F L-C h r 21-2$ & 2016 & Bin 2338 & Bin2339 & & & & 3.19 & 0.28 & 5.65 & & & \\
\hline & & 2016 & Bin2347 & Bin2348 & & & & 2.25 & 0.24 & 4.10 & & & \\
\hline
\end{tabular}


Table 5 (continued)

\begin{tabular}{|c|c|c|c|c|c|c|c|c|c|c|c|c|c|}
\hline \multirow[t]{2}{*}{ Trait } & \multirow[t]{2}{*}{ QTL } & \multirow[t]{2}{*}{ Year } & \multirow{2}{*}{\multicolumn{2}{|c|}{ Flanking markers }} & \multicolumn{3}{|c|}{ Under $\mathrm{E} 1^{\mathrm{c}}$} & \multicolumn{3}{|c|}{ Under E2 } & \multicolumn{3}{|c|}{ Mapping by $D$-value } \\
\hline & & & & & LOD & Effect value & $\operatorname{Var}^{\mathrm{b}}$ & LOD & Effect value & Var\% & LOD & Effect value & Var\% \\
\hline & $q F L-C h r 23-1$ & 2018 & $\operatorname{Bin} 2499$ & $\operatorname{Bin} 2500$ & & & & & & & 3.22 & 0.22 & 6.72 \\
\hline & & 2018 & $\operatorname{Bin} 2503$ & $\operatorname{Bin} 2504$ & 2.11 & 0.24 & 4.73 & & & & & & \\
\hline & $q F L-C h r 23-2$ & 2018 & $\operatorname{Bin} 2521$ & $\operatorname{Bin} 2522$ & 2.83 & -0.33 & 5.93 & & & & & & \\
\hline & $q F L-C h r 24-1$ & 2017 & $\operatorname{Bin} 2558$ & $\operatorname{Bin} 2559$ & 2.67 & 0.26 & 4.74 & & & & & & \\
\hline & $q F L-C h r 24-2$ & 2016 & $\operatorname{Bin} 2581$ & $\operatorname{Bin} 2582$ & & & & 2.66 & 0.25 & 4.52 & & & \\
\hline & & 2016 & $\operatorname{Bin} 2587$ & $\operatorname{Bin} 2588$ & 2.61 & 0.26 & 4.67 & & & & & & \\
\hline & & 2017 & $\operatorname{Bin} 2587$ & $\operatorname{Bin} 2588$ & & & & 3.27 & 0.26 & 5.68 & & & \\
\hline & $q F L-C h r 24-3$ & 2016 & $\operatorname{Bin} 2593$ & $\operatorname{Bin} 2594$ & & & & 4.08 & 0.31 & 6.80 & & & \\
\hline & $q F L-C h r 24-4$ & 2016 & $\operatorname{Bin} 2606$ & $\operatorname{Bin} 2607$ & 2.27 & 0.25 & 4.09 & 2.13 & 0.23 & 3.64 & & & \\
\hline & $q F L-C h r 24-5$ & 2017 & $\operatorname{Bin} 2627$ & $\operatorname{Bin} 2628$ & & & & & & & 2.10 & 0.22 & 4.47 \\
\hline & $q F L-C h r 25-1$ & 2017 & $\operatorname{Bin} 2674$ & $\operatorname{Bin} 2675$ & & & & & & & 2.07 & -0.21 & 4.35 \\
\hline & $q F L-C h r 25-2$ & 2017 & $\operatorname{Bin} 2683$ & $\operatorname{Bin} 2684$ & & & & & & & 4.62 & -0.31 & 10.09 \\
\hline & $q F L-C h r 25-3$ & 2017 & $\operatorname{Bin} 2690$ & $\operatorname{Bin} 2691$ & & & & & & & 4.22 & -0.29 & 8.61 \\
\hline & $q F L-C h r 26-1$ & 2016 & $\operatorname{Bin} 2808$ & $\operatorname{Bin} 2809$ & 2.09 & 0.23 & 3.65 & & & & & & \\
\hline \multirow[t]{30}{*}{$\mathrm{FU}$} & $q F U-C h r 2-1$ & 2018 & Bin110 & Bin111 & & & & & & & 2.88 & -0.33 & 6.12 \\
\hline & $q F U-C h r 4-1$ & 2017 & $\operatorname{Bin} 472$ & $\operatorname{Bin} 473$ & & & & & & & 3.10 & -0.26 & 6.48 \\
\hline & $q F U-C h r 5-1$ & 2017 & Bin492 & Bin493 & & & & 2.66 & -0.20 & 5.18 & & & \\
\hline & $q F U-C h r 5-2$ & 2016 & Bin554 & Bin555 & & & & & & & 2.45 & 0.22 & 4.99 \\
\hline & $q F U-C h r 6-1$ & 2016 & Bin721 & Bin722 & & & & 2.09 & -0.15 & 4.21 & & & \\
\hline & $q F U-C h r 7-1$ & 2017 & Bin775 & Bin776 & & & & 2.04 & -0.17 & 3.79 & & & \\
\hline & $q F U-C h r 7-2$ & 2017 & Bin840 & Bin841 & & & & 2.20 & -0.18 & 4.39 & & & \\
\hline & & 2018 & Bin857 & Bin858 & & & & 2.24 & -0.20 & 4.62 & & & \\
\hline & $q F U-C h r 7-3$ & 2018 & Bin867 & Bin868 & & & & 2.22 & -0.20 & 4.59 & & & \\
\hline & $q F U-C h r 7-4$ & 2018 & Bin881 & Bin882 & & & & 2.05 & -0.19 & 4.25 & & & \\
\hline & $q F U-C h r 9-1$ & 2016 & Bin991 & Bin992 & & & & 3.62 & -0.20 & 6.99 & & & \\
\hline & $q F U-C h r 11-1$ & 2016 & Bin1229 & Bin 1230 & & & & 2.09 & -0.15 & 4.10 & & & \\
\hline & & 2016 & Bin1239 & $\operatorname{Bin} 1240$ & & & & 3.49 & -0.19 & 6.72 & & & \\
\hline & $q F U-C h r 13-1$ & 2016 & Bin1374 & Bin1375 & & & & 4.29 & 0.22 & 8.24 & & & \\
\hline & $q F U-C h r 13-2$ & 2017 & Bin1449 & Bin 1450 & & & & 2.55 & 0.20 & 5.03 & & & \\
\hline & $q F U-C h r 13-3$ & 2018 & Bin1467 & Bin1468 & & & & & & & 2.15 & 0.30 & 4.54 \\
\hline & $q F U-C h r 14-1$ & 2018 & Bin 1520 & $\operatorname{Bin} 1521$ & & & & 3.52 & 0.26 & 8.16 & & & \\
\hline & $q F U-C h r 15-1$ & 2016 & Bin1579 & $\operatorname{Bin} 1580$ & & & & 2.97 & -0.18 & 5.70 & & & \\
\hline & $q F U-C h r 15-2$ & 2016 & Bin1599 & $\operatorname{Bin} 1600$ & & & & & & & 2.65 & 0.23 & 5.54 \\
\hline & $q F U-C h r 15-3$ & 2017 & Bin 1620 & Bin 1621 & & & & 2.25 & 0.19 & 4.84 & & & \\
\hline & & 2017 & $\operatorname{Bin} 1634$ & $\operatorname{Bin} 1635$ & & & & 3.64 & 0.24 & 7.70 & & & \\
\hline & $q F U-C h r 18-1$ & 2016 & Bin1904 & Bin1905 & 2.45 & 0.20 & 5.13 & & & & & & \\
\hline & $q F U-C h r 19-1$ & 2017 & $\operatorname{Bin} 2057$ & $\operatorname{Bin} 2058$ & 2.28 & -0.17 & 4.97 & & & & & & \\
\hline & $q F U-C h r 23-1$ & 2017 & $\operatorname{Bin} 2554$ & $\operatorname{Bin} 2555$ & & & & & & & 2.05 & 0.21 & 4.38 \\
\hline & $q F U-C h r 24-1$ & 2016 & $\operatorname{Bin} 2558$ & $\operatorname{Bin} 2559$ & & & & & & & 3.37 & -0.30 & 6.93 \\
\hline & $q F U-C h r 24-2$ & 2016 & $\operatorname{Bin} 2586$ & $\operatorname{Bin} 2587$ & & & & & & & 2.23 & 0.24 & 4.52 \\
\hline & $q F U-C h r 24-3$ & 2017 & $\operatorname{Bin} 2630$ & $\operatorname{Bin} 2631$ & 2.74 & 0.19 & 5.78 & & & & & & \\
\hline & $q F U-C h r 25-1$ & 2016 & $\operatorname{Bin} 2681$ & $\operatorname{Bin} 2682$ & 2.20 & 0.18 & 4.54 & & & & & & \\
\hline & $q F U-C h r 25-2$ & 2018 & $\operatorname{Bin} 2763$ & $\operatorname{Bin} 2764$ & & & & & & & 2.70 & -0.31 & 5.72 \\
\hline & & 2018 & Bin2774 & $\operatorname{Bin} 2775$ & & & & & & & 2.76 & -0.32 & 5.85 \\
\hline FS & $q F S-C h r l-1$ & 2018 & Bin3 & Bin4 & & & & 2.92 & 0.45 & 6.21 & & & \\
\hline
\end{tabular}


Table 5 (continued)

\begin{tabular}{|c|c|c|c|c|c|c|c|c|c|c|c|c|c|}
\hline \multirow[t]{2}{*}{ Trait } & \multirow[t]{2}{*}{ QTL } & \multirow[t]{2}{*}{ Year } & \multicolumn{2}{|c|}{ Flanking markers } & \multicolumn{3}{|c|}{ Under $\mathrm{E} 1^{\mathrm{c}}$} & \multicolumn{3}{|c|}{ Under E2 } & \multicolumn{3}{|c|}{ Mapping by $D$-value } \\
\hline & & & & & LOD & Effect value & $\operatorname{Var} \%^{\mathrm{b}}$ & LOD & Effect value & $\operatorname{Var} \%$ & LOD & Effect value & Var\% \\
\hline & $q F S-C h r 2-1$ & 2017 & Bin139 & Bin140 & & & & & & & 2.26 & -0.32 & 4.11 \\
\hline & $q F S-C h r 5-1$ & 2016 & $\operatorname{Bin} 488$ & Bin489 & 3.60 & -0.31 & 6.50 & & & & & & \\
\hline & $q F S-C h r 5-2$ & 2016 & $\operatorname{Bin} 500$ & Bin501 & 2.31 & -0.25 & 4.24 & & & & & & \\
\hline & & 2016 & Bin502 & Bin503 & & & & 2.31 & -0.25 & 4.04 & & & \\
\hline & $q F S-C h r 5-3$ & 2017 & Bin548 & Bin549 & 2.22 & -0.33 & 3.90 & & & & & & \\
\hline & $q F S-C h r 5-4$ & 2017 & Bin629 & Bin630 & & & & 2.43 & -0.34 & 4.90 & & & \\
\hline & $q F S-C h r 5-5$ & 2016 & Bin638 & Bin639 & & & & 2.98 & -0.29 & 5.61 & & & \\
\hline & $q F S-C h r 5-6$ & 2017 & Bin640 & Bin641 & 3.98 & -0.45 & 7.06 & & & & & & \\
\hline & $q F S-C h r 5-7$ & 2017 & Bin653 & Bin654 & 5.75 & -0.54 & 9.98 & & & & & & \\
\hline & & 2016 & Bin654 & Bin655 & & & & 5.75 & -0.40 & 10.42 & & & \\
\hline & & 2016 & Bin655 & Bin656 & 2.28 & -0.25 & 4.06 & & & & & & \\
\hline & $q F S-C h r 7-1$ & 2017 & Bin789 & Bin790 & & & & 2.36 & -0.31 & 4.35 & & & \\
\hline & $q F S-C h r 7-2$ & 2017 & Bin804 & Bin805 & & & & & & & 2.98 & 0.37 & 5.82 \\
\hline & $q F S-C h r 7-3$ & 2018 & Bin868 & Bin869 & & & & 2.27 & -0.41 & 5.11 & & & \\
\hline & $q F S-C h r 7-4$ & 2017 & Bin880 & Bin881 & 2.10 & -0.33 & 3.74 & & & & & & \\
\hline & $q F S-C h r 8-1$ & 2018 & Bin912 & Bin913 & & & & & & & 2.27 & -0.37 & 4.85 \\
\hline & $q F S-C h r 8-2$ & 2017 & Bin975 & Bin976 & 3.44 & -0.42 & 6.14 & & & & & & \\
\hline & & 2017 & Bin980 & Bin981 & 2.75 & -0.38 & 5.00 & & & & & & \\
\hline & $q F S-C h r 11-1$ & 2017 & Bin1144 & Bin1145 & & & & & & & 4.05 & -0.44 & 7.95 \\
\hline & $q F S-C h r 14-1$ & 2016 & Bin1501 & Bin1502 & & & & 3.08 & 0.30 & 5.42 & & & \\
\hline & $q F S-C h r 15-1$ & 2017 & Bin1659 & Bin 1660 & & & & 2.14 & 0.29 & 3.73 & & & \\
\hline & $q F S-C h r 17-1$ & 2017 & Bin1861 & Bin1862 & & & & & & & 2.95 & -0.44 & 5.75 \\
\hline & $q F S-C h r 17-2$ & 2016 & Bin 1870 & Bin1871 & & & & & & & 2.06 & 0.29 & 4.36 \\
\hline & $q F S-C h r 18-1$ & 2017 & Bin1909 & Bin1910 & & & & & & & 3.33 & 0.39 & 6.51 \\
\hline & $q F S-C h r 19-1$ & 2017 & Bin2066 & Bin2067 & & & & & & & 2.75 & 0.36 & 5.35 \\
\hline & $q F S-C h r 22-1$ & 2018 & $\operatorname{Bin} 2411$ & Bin2412 & & & & & & & 2.72 & 0.52 & 6.64 \\
\hline & $q F S-C h r 24-1$ & 2017 & $\operatorname{Bin} 2578$ & $\operatorname{Bin} 2579$ & 6.25 & 0.56 & 11.19 & & & & & & \\
\hline & & 2017 & Bin2579 & $\operatorname{Bin} 2580$ & & & & 2.90 & 0.35 & 5.39 & & & \\
\hline & & 2016 & Bin2581 & $\operatorname{Bin} 2582$ & & & & 2.60 & 0.27 & 4.66 & & & \\
\hline & & 2016 & $\operatorname{Bin} 2584$ & $\operatorname{Bin} 2585$ & 4.06 & 0.35 & 7.94 & & & & & & \\
\hline & $q F S-C h r 24-2$ & 2016 & Bin 2593 & $\operatorname{Bin} 2594$ & 6.83 & 0.44 & 12.90 & & & & & & \\
\hline & & 2017 & Bin2592 & Bin 2593 & 7.19 & 0.60 & 12.73 & & & & & & \\
\hline & & 2017 & Bin2596 & Bin2597 & & & & 2.74 & 0.34 & 5.10 & & & \\
\hline & $q F S-C h r 24-3$ & 2017 & $\operatorname{Bin} 2601$ & Bin2602 & 4.78 & 0.51 & 8.72 & & & & & & \\
\hline & $q F S-C h r 25-1$ & 2016 & $\operatorname{Bin} 2707$ & $\operatorname{Bin} 2708$ & 2.03 & -0.24 & 3.66 & & & & & & \\
\hline & & 2016 & Bin2712 & $\operatorname{Bin} 2713$ & 2.67 & -0.28 & 4.78 & & & & & & \\
\hline & $q F S-C h r 25-2$ & 2016 & $\operatorname{Bin} 2769$ & $\operatorname{Bin} 2770$ & 3.21 & 0.30 & 5.79 & & & & 2.17 & 0.27 & 4.95 \\
\hline \multirow[t]{9}{*}{ FE } & $q F E-C h r 2-1$ & 2017 & Bin128 & Bin129 & & & & & & & 2.95 & -0.02 & 6.44 \\
\hline & $q F E-C h r 5-1$ & 2016 & Bin493 & Bin494 & & & & 5.49 & -0.03 & 11.13 & & & \\
\hline & $q F E-C h r 5-2$ & 2016 & $\operatorname{Bin} 521$ & Bin522 & & & & 3.26 & 0.02 & 6.42 & & & \\
\hline & $q F E-C h r 5-3$ & 2016 & Bin530 & Bin531 & & & & 2.13 & 0.02 & 4.36 & & & \\
\hline & $q F E-C h r 7-1$ & 2018 & Bin872 & Bin873 & & & & 3.33 & -0.01 & 6.80 & & & \\
\hline & & 2018 & Bin879 & Bin880 & & & & & & & 5.28 & 0.02 & 11.19 \\
\hline & $q F E-C h r 9-1$ & 2017 & Bin 1010 & Bin1011 & & & & 3.18 & -0.02 & 6.10 & & & \\
\hline & $q F E-C h r 10-1$ & 2017 & Bin1123 & Bin1124 & 3.04 & 0.02 & 5.98 & & & & & & \\
\hline & $q F E-C h r 10-2$ & 2017 & Bin1132 & Bin1133 & 3.72 & 0.02 & 7.44 & & & & & & \\
\hline
\end{tabular}


Table 5 (continued)

\begin{tabular}{|c|c|c|c|c|c|c|c|c|c|c|c|c|c|}
\hline \multirow[t]{2}{*}{ Trait } & \multirow[t]{2}{*}{ QTL } & \multirow[t]{2}{*}{ Year } & \multicolumn{2}{|c|}{ Flanking markers } & \multicolumn{3}{|c|}{ Under $\mathrm{E} 1^{\mathrm{c}}$} & \multicolumn{3}{|c|}{ Under E2 } & \multicolumn{3}{|c|}{ Mapping by $D$-value } \\
\hline & & & & & LOD & Effect value & $\operatorname{Var}^{\mathrm{b}}$ & LOD & Effect value & Var\% & LOD & Effect value & $\operatorname{Var} \%$ \\
\hline & $q F E-C h r 11-1$ & 2018 & Bin1192 & Bin1193 & & & & 3.16 & -0.01 & 6.43 & & & \\
\hline & $q F E-C h r 12-1$ & 2016 & Bin1364 & Bin1365 & 2.08 & 0.02 & 4.65 & & & & & & \\
\hline & $q F E-C h r 14-1$ & 2018 & Bin1494 & Bin1495 & 3.65 & -0.01 & 7.66 & & & & & & \\
\hline & $q F E-C h r 14-2$ & 2018 & Bin 1508 & Bin 1509 & 4.60 & -0.01 & 9.54 & & & & & & \\
\hline & & 2018 & Bin 1516 & Bin 1517 & 3.17 & -0.01 & 6.68 & & & & & & \\
\hline & $q F E-C h r 14-3$ & 2018 & Bin 1553 & Bin 1554 & & & & 2.25 & 0.01 & 4.60 & & & \\
\hline & $q F E-C h r 15-1$ & 2016 & Bin 1589 & Bin 1590 & 2.04 & 0.02 & 4.52 & & & & & & \\
\hline & & 2016 & Bin1596 & Bin 1597 & & & & & & & 2.80 & 0.02 & 5.68 \\
\hline & & 2016 & Bin 1599 & Bin 1600 & 2.08 & 0.02 & 4.22 & & & & & & \\
\hline & qFE-Chrl6-1 & 2016 & Bin 1718 & Bin1719 & & & & 2.94 & -0.02 & 5.94 & & & \\
\hline & $q F E-C h r 17-1$ & 2017 & Bin 1835 & Bin 1836 & & & & 2.17 & 0.02 & 4.35 & & & \\
\hline & $q F E-C h r l 8-1$ & 2018 & Bin1971 & Bin1972 & & & & 3.04 & -0.01 & 6.19 & & & \\
\hline & $q F E-C h r 19-1$ & 2018 & Bin2089 & $\operatorname{Bin} 2090$ & & & & 2.42 & -0.01 & 4.87 & & & \\
\hline & $q F E-C h r 19-2$ & 2018 & Bin2099 & $\operatorname{Bin} 2100$ & & & & 2.37 & -0.01 & 4.78 & & & \\
\hline & $q F E-C h r 20-1$ & 2017 & Bin 2185 & $\operatorname{Bin} 2186$ & & & & 3.76 & -0.02 & 7.13 & & & \\
\hline & $q F E-C h r 20-2$ & 2018 & $\operatorname{Bin} 2204$ & $\operatorname{Bin} 2204$ & 2.05 & 0.01 & 3.90 & & & & & & \\
\hline & $q F E-C h r 20-3$ & 2016 & $\operatorname{Bin} 2248$ & $\operatorname{Bin} 2249$ & & & & & & & 4.64 & 0.03 & 10.43 \\
\hline & & 2016 & $\operatorname{Bin} 2254$ & $\operatorname{Bin} 2255$ & & & & & & & 3.62 & 0.02 & 7.41 \\
\hline & & 2017 & $\operatorname{Bin} 2267$ & $\operatorname{Bin} 2268$ & & & & 2.07 & 0.01 & 3.63 & & & \\
\hline & $q F E-C h r 21-1$ & 2018 & Bin 2279 & $\operatorname{Bin} 2280$ & & & & & & & 4.21 & -0.02 & 8.83 \\
\hline & $q F E-C h r 21-2$ & 2017 & Bin2336 & $\operatorname{Bin} 2337$ & & & & 2.56 & 0.02 & 4.76 & & & \\
\hline & $q F E-C h r 21-3$ & 2017 & $\operatorname{Bin} 2345$ & $\operatorname{Bin} 2346$ & 2.28 & 0.02 & 4.37 & & & & & & \\
\hline & $q F E-C h r 22-1$ & 2017 & $\operatorname{Bin} 2393$ & $\operatorname{Bin} 2394$ & & & & & & & 2.90 & 0.02 & 5.96 \\
\hline & $q F E-C h r 22-2$ & 2017 & $\operatorname{Bin} 2412$ & $\operatorname{Bin} 2413$ & & & & 2.55 & 0.02 & 4.44 & & & \\
\hline & $q F E-C h r 24-1$ & 2018 & $\operatorname{Bin} 2584$ & $\operatorname{Bin} 2585$ & 3.70 & 0.01 & 7.59 & & & & & & \\
\hline & $q F E-C h r 25-1$ & 2017 & $\operatorname{Bin} 2695$ & $\operatorname{Bin} 2696$ & & & & & & & 2.90 & -0.02 & 5.95 \\
\hline \multirow[t]{14}{*}{ FM } & $q F M-C h r 1-1$ & 2016 & Bin96 & Bin97 & & & & 3.13 & 0.10 & 6.03 & & & \\
\hline & $q F M-C h r 2-1$ & 2017 & Bin128 & Bin129 & & & & 2.71 & -0.06 & 4.02 & & & \\
\hline & $q F M-C h r 4-1$ & 2018 & $\operatorname{Bin} 360$ & Bin361 & & & & 2.75 & 0.08 & 5.74 & & & \\
\hline & & 2018 & $\operatorname{Bin} 366$ & Bin367 & & & & 2.18 & 0.07 & 4.58 & & & \\
\hline & $q F M-C h r 4-2$ & 2017 & $\operatorname{Bin} 484$ & Bin 485 & & & & 3.99 & -0.07 & 5.72 & & & \\
\hline & $q F M-C h r 5-1$ & 2017 & Bin 488 & Bin489 & & & & 2.21 & 0.05 & 2.91 & & & \\
\hline & $q F M-C h r 5-2$ & 2017 & Bin641 & Bin642 & & & & 6.00 & 0.09 & 9.37 & & & \\
\hline & & 2017 & Bin645 & Bin646 & 4.85 & 0.09 & 7.98 & 8.35 & 0.10 & 12.66 & & & \\
\hline & $q F M-C h r 5-3$ & 2017 & Bin655 & Bin656 & 6.06 & 0.10 & 9.81 & & & & & & \\
\hline & & 2017 & Bin657 & Bin658 & & & & 7.24 & 0.10 & 11.13 & & & \\
\hline & $q F M-C h r 7-1$ & 2016 & Bin842 & Bin843 & & & & 2.53 & 0.08 & 4.51 & & & \\
\hline & & 2016 & Bin850 & Bin851 & & & & 2.16 & 0.08 & 3.95 & & & \\
\hline & $q F M-C h r 8-1$ & 2018 & Bin910 & Bin911 & 2.84 & 0.08 & 5.85 & & & & & & \\
\hline & $q F M-C h r 8-2$ & 2018 & Bin919 & Bin920 & 2.99 & 0.08 & 6.15 & 2.88 & 0.08 & 6.02 & & & \\
\hline
\end{tabular}


Table 5 (continued)

\begin{tabular}{|c|c|c|c|c|c|c|c|c|c|c|c|c|c|}
\hline \multirow[t]{2}{*}{ Trait } & \multirow[t]{2}{*}{ QTL } & \multirow[t]{2}{*}{ Year } & \multicolumn{2}{|c|}{ Flanking markers } & \multicolumn{3}{|c|}{${\text { Under } \mathrm{E} 1^{\mathrm{c}}}$} & \multicolumn{3}{|c|}{ Under E2 } & \multicolumn{3}{|c|}{ Mapping by $D$-value } \\
\hline & & & & & LOD & Effect value & $\operatorname{Var} \%^{\mathrm{b}}$ & LOD & Effect value & Var\% & LOD & Effect value & Var\% \\
\hline & \multirow[t]{6}{*}{$q F M-C h r 8-3 *$} & 2017 & Bin964 & Bin965 & 2.73 & 0.06 & 4.04 & & & & & & \\
\hline & & 2017 & Bin977 & Bin978 & 2.02 & 0.06 & 3.01 & & & & & & \\
\hline & & 2016 & Bin969 & Bin970 & & & & \multirow[t]{2}{*}{3.48} & \multirow[t]{2}{*}{0.10} & \multirow[t]{2}{*}{6.71} & & & \\
\hline & & 2016 & Bin970 & Bin971 & 2.94 & 0.09 & 5.48 & & & & & & \\
\hline & & 2018 & Bin977 & Bin978 & 2.34 & -0.07 & 4.85 & \multirow[t]{2}{*}{3.46} & -0.09 & \multirow[t]{2}{*}{7.30} & & & \\
\hline & & 2018 & Bin982 & Bin983 & 2.84 & -0.07 & 5.86 & & & & & & \\
\hline & $q F M-C h r 13-1$ & 2018 & Bin1434 & Bin1435 & & & & & & & 2.49 & 0.05 & 5.41 \\
\hline & $q F M-C h r 13-2$ & 2018 & Bin1456 & Bin1457 & & & & & & & 2.03 & 0.04 & 4.44 \\
\hline & $q F M-C h r l 3-3$ & 2016 & Bin1473 & Bin1474 & 2.20 & 0.09 & 3.84 & & & & & & \\
\hline & qFM-Chr14-1 & 2017 & Bin1502 & Bin 1503 & 3.11 & -0.07 & 4.59 & & & & & & \\
\hline & \multirow[t]{2}{*}{$q F M-C h r 14-2$} & 2016 & Bin1521 & Bin1522 & & & & & & & 2.54 & -0.10 & 5.57 \\
\hline & & 2016 & Bin 1528 & Bin1529 & & & & & & & 2.37 & -0.09 & 4.99 \\
\hline & $q F M-C h r 14-3$ & 2016 & Bin1540 & Bin1541 & 3.87 & -0.11 & 8.16 & & & & & & \\
\hline & $q F M-C h r 14-4$ & 2016 & Bin 1550 & Bin1551 & 4.63 & -0.12 & 8.82 & & & & & & \\
\hline & \multirow[t]{6}{*}{$q F M-C h r 15-1 *$} & 2016 & Bin1568 & Bin1569 & & & & 2.08 & -0.08 & 4.11 & & & \\
\hline & & 2016 & Bin1579 & Bin1580 & & & & 3.84 & -0.11 & 7.42 & & & \\
\hline & & 2016 & Bin1580 & Bin1581 & & & & & & & 3.38 & 0.11 & 7.22 \\
\hline & & 2016 & Bin1589 & Bin 1590 & & & & 2.48 & -0.09 & 4.88 & 2.42 & 0.10 & 6.45 \\
\hline & & 2018 & Bin1571 & Bin 1572 & & & & & & & 3.55 & -0.06 & 7.37 \\
\hline & & 2018 & Bin1594 & Bin1595 & & & & & & & 4.94 & 0.07 & 10.49 \\
\hline & $q F M-C h r 15-2$ & 2017 & Bin1678 & Bin1679 & & & & 2.02 & -0.05 & 2.84 & & & \\
\hline & \multirow[t]{3}{*}{$q F M-C h r 15-3$} & 2016 & Bin1690 & Bin1691 & & & & 3.48 & -0.10 & 6.72 & & & \\
\hline & & 2017 & Bin1690 & Bin1691 & & & & 2.48 & -0.06 & 3.84 & & & \\
\hline & & 2017 & Bin1689 & Bin1690 & 5.53 & -0.10 & 9.13 & & & & & & \\
\hline & \multirow[t]{2}{*}{$q F M-C h r 16-1$} & 2016 & Bin1817 & Bin1818 & & & & & & & 2.03 & -0.09 & 4.38 \\
\hline & & 2016 & Bin1821 & Bin1822 & & & & 2.11 & 0.08 & 3.92 & 3.10 & -0.11 & 6.59 \\
\hline & $q F M-C h r 19-1$ & 2017 & Bin2061 & Bin2062 & & & & & & & 3.00 & -0.05 & 6.15 \\
\hline & $q F M-C h r 19-2$ & 2017 & Bin2073 & Bin2074 & & & & & & & 2.37 & -0.04 & 4.88 \\
\hline & \multirow[t]{3}{*}{$q F M-C h r 21-1$} & 2017 & Bin2279 & $\operatorname{Bin} 2280$ & & & & 2.05 & -0.05 & 2.71 & & & \\
\hline & & 2016 & $\operatorname{Bin} 2285$ & $\operatorname{Bin} 2286$ & 2.00 & 0.08 & 3.77 & & & & & & \\
\hline & & 2016 & Bin2292 & Bin2293 & 2.77 & 0.09 & 5.15 & & & & & & \\
\hline & $q F M-C h r 21-2$ & 2017 & $\operatorname{Bin} 2330$ & Bin2331 & & & & & & & 2.75 & 0.06 & 5.77 \\
\hline & $q F M-C h r 22-1$ & 2017 & Bin2464 & $\operatorname{Bin} 2465$ & 3.11 & -0.07 & 4.97 & & & & & & \\
\hline & \multirow[t]{2}{*}{$q F M-C h r 22-2$} & 2017 & Bin 2472 & Bin2473 & & & & 10.29 & -0.11 & 16.03 & & & \\
\hline & & 2017 & $\operatorname{Bin} 2473$ & $\operatorname{Bin} 2474$ & 2.44 & -0.06 & 3.94 & & & & & & \\
\hline & $q F M-C h r 23-1$ & 2017 & $\operatorname{Bin} 2537$ & $\operatorname{Bin} 2538$ & & & & 2.06 & 0.05 & 2.73 & & & \\
\hline & $q F M-C h r 25-1$ & 2016 & $\operatorname{Bin} 2700$ & $\operatorname{Bin} 2701$ & 2.48 & 0.09 & 4.91 & & & & 2.06 & 0.08 & 4.32 \\
\hline & & 2016 & Bin2706 & Bin2707 & 2.47 & 0.08 & 4.56 & & & & & & \\
\hline & $q F M-C h r 25-2$ & 2018 & Bin2726 & $\operatorname{Bin} 2727$ & & & & 2.07 & -0.07 & 4.42 & & & \\
\hline & $q F M-C h r 25-3$ & 2018 & $\operatorname{Bin} 2738$ & Bin2739 & & & & 3.29 & -0.09 & 6.92 & & & \\
\hline & $q F M-C h r 25-4$ & 2018 & Bin2751 & Bin2752 & & & & 2.05 & -0.07 & 4.39 & & & \\
\hline & $q F M-C h r 26-1$ & 2016 & $\operatorname{Bin} 2810$ & Bin2811 & & & & 2.37 & -0.08 & 4.52 & & & \\
\hline & $q F M-C h r 26-2$ & 2016 & Bin 2837 & Bin 2838 & 4.48 & -0.12 & 8.74 & & & & & & \\
\hline & $q F M-C h r 26-3$ & 2016 & $\operatorname{Bin} 2845$ & $\operatorname{Bin} 2846$ & 5.55 & -0.13 & 10.69 & & & & & & \\
\hline
\end{tabular}

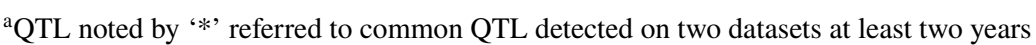

${ }^{\mathrm{b}}$ Phenotypic variation explained by a single locus QTL

${ }^{\mathrm{c}} \mathrm{E} 1$, salt stress condition; E2, normal condition; $D$-value, the difference values between salt stress and normal conditions 


\section{Pleiotropic effects analysis on FL and FS}

A total of 35 clusters located on 18 chromosomes showed pleiotropic effects involving 107 QTL (Table 6). Of these, four clusters existed on chromosome (Chr) 5, Chr7, and Chr24; three were detected on Chr15 and Chr25; two were detected on Chr8, Chr14, Chr16, and Chr21, and only one cluster was detected on other 9 chromosomes.

There are two pleiotropic regions that can improve five fiber quality traits, which are Loci-Chr5-1 and LociChr14-1, respectively. Two clusters could improve four fiber quality traits, which including Loci-Chr15-1 (FL, FU, FE FM), Loci-Chr24-2 (FL, FU, FE, FS). Six clusters controlled three traits, which were Loci-Chr5-4 (FL, FS, FM), LociChr7-4 (FU FS, FE), Loci-Chr19-1 (FU, FS, FM), LociChr21-2 (FL, FE, FM), Loci-Chr23-1 (FL, FE, FM), and Loci-Chr25-2 (FS, FE, FM), respectively.

Among the 35 pleiotropic regions, six affected FL and FS simultaneously, which were distributed on Chr5, Chr7, $\mathrm{Chr}$ 14, and Chr24, of which two regions were found in both Chr5 and Chr24. Loci-Chr5-1, Loci-Chr5-4, and LociChr7-1, controlled FL and FS simultaneously and received favorable alleles contributed by the female GX1135. Among them, the two QTL $q F S$-Chr5-1 and $q F S$-Chr5-2 that controlled FS on Loci-Chr5-1 were detected in E1 and E2 in 2016, and the two QTL $q F L-C h r 5-1$ and $q F L-C h r 5-2$ that control FL were detected in E1 and E2 in 2016 and 2017 simultaneously. The cluster also controlled FU, FE, and FM. The Loci-Chr5-4 cluster contains six QTL, of which three QTL controlled FS ( $q F S-C h r 5-5, q F S-C h r 5-6$ and $q F S$ Chr5-7), one controlled FL ( $q F L-C h r 5-5)$, which detected in E1 and E2 in 2016 and 2017, and the region also controls FM ( $q F M-C h r 5-2, q F M-C h r 5-3)$. Two QTL were detected on Loci-Chr7-1, of which, $q F S$-Chr7-1, affecting FS was detected in E2 in 2017, but $q F L-C h r 7-1$ was detected in E3 in 2016. Loci-Chr24-2 and Loci-Chr24-3, two pleiotropic regions on chromosome 24, were detected in both E1 and E2 in 2016 and 2017, and the favorable alleles were contributed by male parent GX100-2. Among them, two QTL influencing FL and FS in Loci-Chr24-2, (qFS-Chr24-1, $q F S-C h r 24-2$ and $q F L-C h r 24-2, q F L-C h r 24-3)$, which also control FE and FU simultaneously. Eight QTL were detected in Loci-Chr14-1, of which only one QTL ( $q$ FS-Chr14-1) influencing FS was detected in E2, 2016, two QTL from Loci-Chr14-1 influencing FL were detected in E2, 2018. The favorable alleles in $q F S$-Chrl4- 1 and $q F L-C h r 14-2$ originated from the female GX1135, while the favorable alleles in $q F L-C h r 14-3$ were contributed by male GX100-2.

\section{Identification of candidate genes within QTL and expression validation by RNA-seq}

In this study, two QTL ( $q F L-C h r 1-1$ and $q F L-C h r 5-5$ ) influencing FL were detected in both E1 and E2 and detected repeatedly across two years. Explaining $4.26 \%$ of the average PV, $q F L-C h r l-1$, is favorably provided by the female parent. The QTL, $q F L-C h r 5-5$, is provided by male parent, and the average PV is $9.38 \%$. Among them, the confidence interval of $q F L-C h r 1-1$ is between marker Bin 28 and Bin29, corresponding to the reference genome of upland cotton TM-1 $10,876,918$ bp to $11,016,009 \mathrm{bp}$, the physical distance of this interval is $139,091 \mathrm{bp}$, which includes 12 annotated genes (Gh_A01G0604-Gh_A01G0615). The flanking markers of $q F L-C h r 5-5$ are Bin648 and Bin649, corresponding to the reference genome of upland cotton $88,623,793$ bp to $88,671,394 \mathrm{bp}$, the physical distance of this region is $47,601 \mathrm{bp}$, including two annotated genes (Gh_A05G3395 and Gh_A05G3396) (Table 7). Among them, there are 11 known genes and three genes were with uncharacterized protein information. The known genes were annotated as Microtubule-associated protein TORTIFOLIA1, DNA polymerase I A, UDP-glycosyltransferase 73C5, Serine/threonine-protein kinase PBS1, Vacuolar protein sorting-associated protein, Fatty acid desaturase 4, Cytochrome P450 716B1, Protein FATTY ACID EXPORT 5, Polyadenylate-binding protein RBP45C, Probable protein phosphatase 2C 33, and Aspartic proteinase-like protein 2, respectively. These putative candidate genes were explored for the public RNA-seq expression data of TM-1 in fiber development (Fig. 1). The result showed that fragments per kilobase million (FPKM) of four genes (Gh_A01G0606, $G h \_A 01 G 0607, G h \_A 01 G 0611$, and Gh_A01G0613) were less than 1 . The gene (Gh_A01G0615) is persistently highly expressed at different stages of fiber development. Three genes (Gh_A01G0604, Gh_A01G0605, and Gh_A01G3396) were highly expressed at various stages of fiber development $(5,10$, and 20 DPA $)$.

To validate the potential function in fiber development, the expression pattern of candidate genes was verified with qRT-PCR using fibers of extremely long fiber line $(\mathrm{H})$, extremely short fiber line (L), female (GX1135, F), male (GX100-2, M) at different developmental stages (Fig. 2). The expression of Gh_A01G0604 in extremely long fiber line was six times higher than that in extremely short fiber line at 5 DPA. The expression of Gh_A01G0610, Gh_A01G0612, Gh_A01G0615, Gh_A01G3395, and Gh_ A01G3396 in extremely long fiber line was significantly higher than that of extremely short fiber line at many points from 10 to 20 DPA. Therefore, these seven genes were identified as candidate genes responsible for influencing fiber cell elongation. 
Table 6 Pleiotropic regions in the present study for fiber quality traits

\begin{tabular}{|c|c|c|c|c|c|c|c|c|c|c|c|c|c|}
\hline \multirow[t]{2}{*}{ Cluster } & \multirow[t]{2}{*}{ QTL } & \multirow[t]{2}{*}{ Year } & \multicolumn{2}{|c|}{ Flanking markers } & \multicolumn{3}{|c|}{ Under $\mathrm{E} 1^{\mathrm{c}}$} & \multicolumn{3}{|c|}{ Under E2 } & \multicolumn{3}{|c|}{ Mapping by $D$-value } \\
\hline & & & & & LOD & Additive & $\operatorname{Var} \%{ }^{\mathrm{b}}$ & LOD & Additive & Var\% & LOD & Additive & $\operatorname{Var} \%$ \\
\hline \multirow[t]{3}{*}{ Loci-Chr2-1 } & $q F E-C h r 2-1$ & 2017 & Bin128 & Bin129 & & & & & & & 2.95 & -0.02 & 6.44 \\
\hline & $q F M-C h r 2-1$ & 2017 & Bin128 & Bin129 & & & & 2.71 & -0.06 & 4.02 & & & \\
\hline & $q F S-C h r 2-1$ & 2017 & Bin139 & Bin140 & & & & & & & 2.26 & -0.32 & 4.11 \\
\hline \multirow[t]{2}{*}{ Loci-Chr4-1 } & $q F U-C h r 4-1$ & 2017 & Bin472 & Bin473 & & & & & & & 3.10 & -0.26 & 6.48 \\
\hline & $q F M-C h r 4-2$ & 2017 & Bin484 & Bin485 & & & & 3.99 & -0.07 & 5.72 & & & \\
\hline \multirow[t]{10}{*}{ Loci-Chr5-1 } & $q F M-C h r 5-1$ & 2017 & $\operatorname{Bin} 488$ & Bin489 & & & & 2.21 & 0.05 & 2.91 & & & \\
\hline & $q F U-C h r 5-1$ & 2017 & Bin492 & Bin493 & & & & 2.66 & -0.20 & 5.18 & & & \\
\hline & $q F E-C h r 5-1$ & 2016 & Bin493 & Bin494 & & & & 5.49 & -0.03 & 11.13 & & & \\
\hline & $q F S-C h r 5-1$ & 2016 & Bin488 & Bin489 & 3.60 & -0.31 & 6.50 & & & & & & \\
\hline & $q F S-C h r 5-2$ & 2016 & Bin500 & Bin501 & 2.31 & -0.25 & 4.24 & & & & & & \\
\hline & & 2016 & Bin502 & Bin503 & & & & 2.31 & -0.25 & 4.04 & & & \\
\hline & $q F L-C h r 5-2$ & 2016 & Bin500 & Bin501 & 2.79 & -0.26 & 4.87 & & & & & & \\
\hline & $q F L-C h r 5-1$ & 2016 & Bin490 & Bin491 & & & & 4.61 & -0.34 & 8.20 & & & \\
\hline & & 2017 & Bin493 & Bin494 & 6.49 & -0.40 & 12.11 & & & & & & \\
\hline & $q F L-C h r 5-2$ & 2017 & Bin502 & Bin503 & 5.08 & -0.36 & 9.66 & & & & & & \\
\hline \multirow[t]{5}{*}{ Loci-Chr5-2 } & $q F L-C h r 5-3$ & 2016 & Bin512 & Bin513 & & & & 5.87 & -0.37 & 10.31 & & & \\
\hline & & 2017 & Bin515 & Bin516 & & & & 6.23 & -0.36 & 11.06 & & & \\
\hline & $q F L-C h r 5-4$ & 2017 & Bin527 & Bin528 & & & & 6.90 & -0.38 & 12.14 & & & \\
\hline & $q F E-C h r 5-2$ & 2016 & Bin521 & Bin522 & & & & 3.26 & 0.02 & 6.42 & & & \\
\hline & $q F E-C h r 5-3$ & 2016 & Bin530 & Bin531 & & & & 2.13 & 0.02 & 4.36 & & & \\
\hline \multirow[t]{2}{*}{ Loci-Chr5-3 } & $q F S-C h r 5-3$ & 2017 & Bin548 & Bin549 & 2.22 & -0.33 & 3.90 & & & & & & \\
\hline & $q F U-C h r 5-2$ & 2016 & Bin554 & Bin555 & & & & & & & 2.45 & 0.22 & 4.99 \\
\hline \multirow[t]{11}{*}{ Loci-Chr5-4 } & $q F S-C h r 5-5$ & 2016 & Bin638 & Bin639 & & & & 2.98 & -0.29 & 5.61 & & & \\
\hline & $q F S-C h r 5-6$ & 2017 & Bin640 & Bin641 & 3.98 & -0.45 & 7.06 & & & & & & \\
\hline & $q F S-C h r 5-7$ & 2017 & Bin653 & Bin654 & 5.75 & -0.54 & 9.98 & & & & & & \\
\hline & & 2016 & Bin654 & Bin655 & & & & 5.75 & -0.40 & 10.42 & & & \\
\hline & & 2016 & Bin655 & Bin656 & 2.28 & -0.25 & 4.06 & & & & & & \\
\hline & $q F L-C h r 5-5^{* a}$ & 2016 & Bin648 & Bin649 & 5.91 & -0.39 & 10.80 & 6.73 & -0.40 & 11.65 & & & \\
\hline & & 2017 & Bin648 & Bin649 & & & & 3.29 & -0.26 & 5.68 & & & \\
\hline & $q F M-C h r 5-2 *$ & 2017 & Bin641 & Bin642 & & & & 6.00 & 0.09 & 9.37 & & & \\
\hline & & 2017 & Bin645 & Bin646 & 4.85 & 0.09 & 7.98 & 8.35 & 0.10 & 12.66 & & & \\
\hline & $q F M-C h r 5-3$ & 2017 & Bin655 & Bin656 & 6.06 & 0.10 & 9.81 & & & & & & \\
\hline & & 2017 & Bin657 & Bin658 & & & & 7.24 & 0.10 & 11.13 & & & \\
\hline \multirow[t]{2}{*}{ Loci-Chr7-1 } & $q F S-C h r 7-1$ & 2017 & Bin789 & Bin790 & & & & 2.36 & -0.31 & 4.35 & & & \\
\hline & $q F L-C h r 7-1$ & 2016 & Bin795 & Bin796 & & & & & & & 2.49 & 0.23 & 5.57 \\
\hline \multirow[t]{4}{*}{ Loci-Chr7-2 } & $q F M-C h r 7-1$ & 2016 & Bin842 & Bin843 & & & & 2.53 & 0.08 & 4.51 & & & \\
\hline & & 2016 & Bin850 & Bin851 & & & & 2.16 & 0.08 & 3.95 & & & \\
\hline & $q F U-C h r 7-2$ & 2017 & Bin840 & Bin841 & & & & 2.20 & -0.18 & 4.39 & & & \\
\hline & & 2018 & Bin857 & Bin858 & & & & 2.24 & -0.20 & 4.62 & & & \\
\hline \multirow[t]{2}{*}{ Loci-Chr7-3 } & $q F U-C h r 7-3$ & 2018 & Bin867 & Bin868 & & & & 2.22 & -0.20 & 4.59 & & & \\
\hline & $q F S-C h r 7-3$ & 2018 & Bin868 & Bin869 & & & & 2.27 & -0.41 & 5.11 & & & \\
\hline \multirow[t]{4}{*}{ Loci-Chr7-4 } & $q F E-C h r 7-1$ & 2018 & Bin872 & Bin873 & & & & 3.33 & -0.01 & 6.80 & & & \\
\hline & & 2018 & Bin879 & Bin880 & & & & & & & 5.28 & 0.02 & 11.19 \\
\hline & $q F S-C h r 7-4$ & 2017 & Bin880 & Bin881 & 2.10 & -0.33 & 3.74 & & & & & & \\
\hline & $q F U-C h r 7-4$ & 2018 & Bin881 & Bin882 & & & & 2.05 & -0.19 & 4.25 & & & \\
\hline \multirow[t]{2}{*}{ Loci-Chr8-1 } & $q F M-C h r 8-1$ & 2018 & Bin910 & Bin911 & 2.84 & 0.08 & 5.85 & & & & & & \\
\hline & $q F S-C h r 8-1$ & 2018 & Bin912 & Bin913 & & & & & & & 2.27 & -0.37 & 4.85 \\
\hline
\end{tabular}


Table 6 (continued)

\begin{tabular}{|c|c|c|c|c|c|c|c|c|c|c|c|c|c|}
\hline \multirow[t]{2}{*}{ Cluster } & \multirow[t]{2}{*}{ QTL } & \multirow[t]{2}{*}{ Year } & \multicolumn{2}{|c|}{ Flanking markers } & \multicolumn{3}{|c|}{ Under $\mathrm{E} 1^{\mathrm{c}}$} & \multicolumn{3}{|c|}{ Under E2 } & \multicolumn{3}{|c|}{ Mapping by $D$-value } \\
\hline & & & & & LOD & Additive & $\operatorname{Var} \%^{\mathrm{b}}$ & LOD & Additive & $\operatorname{Var} \%$ & LOD & Additive & Var\% \\
\hline \multirow[t]{9}{*}{ Loci-Chr8-2 } & $q F M-C h r 8-3^{*}$ & 2017 & Bin964 & Bin965 & 2.73 & 0.06 & 4.04 & & & & & & \\
\hline & & 2016 & Bin969 & Bin970 & & & & 3.48 & 0.10 & 6.71 & & & \\
\hline & & 2016 & Bin970 & Bin971 & & & & 2.94 & 0.09 & 5.48 & & & \\
\hline & & 2018 & Bin977 & Bin978 & & & & 2.34 & -0.07 & 4.85 & & & \\
\hline & & 2018 & Bin977 & Bin978 & & & & 3.46 & -0.09 & 7.30 & & & \\
\hline & & 2017 & Bin977 & Bin978 & 2.02 & 0.06 & 3.01 & & & & & & \\
\hline & & 2018 & Bin982 & Bin983 & 2.84 & -0.07 & 5.86 & & & & & & \\
\hline & $q F S-C h r 8-2$ & 2017 & Bin975 & Bin976 & 3.44 & -0.42 & 6.14 & & & & & & \\
\hline & & 2017 & Bin980 & Bin981 & 2.75 & -0.38 & 5.00 & & & & & & \\
\hline \multirow[t]{3}{*}{ Loci-Chr12-1 } & $q F E-C h r 12-1$ & 2016 & Bin1364 & Bin1365 & 2.08 & 0.02 & 4.65 & & & & & & \\
\hline & $q F L-C h r 12-1$ & 2018 & Bin1368 & Bin1369 & & & & & & & 2.97 & -0.22 & 6.17 \\
\hline & & 2018 & Bin1371 & Bin1372 & & & & 2.72 & 0.27 & 5.45 & & & \\
\hline \multirow[t]{4}{*}{ Loci-Chr13-1 } & $q F U-C h r 13-2$ & 2017 & Bin1449 & Bin1450 & & & & 2.55 & 0.20 & 5.03 & & & \\
\hline & $q F U-C h r 13-3$ & 2018 & Bin1467 & Bin1468 & & & & & & & 2.15 & 0.30 & 4.54 \\
\hline & $q F M-C h r 13-2$ & 2018 & Bin 1456 & Bin1457 & & & & & & & 2.03 & 0.04 & 4.44 \\
\hline & $q F M-C h r 13-3$ & 2016 & Bin 1473 & Bin1474 & 2.20 & 0.09 & 3.84 & & & & & & \\
\hline \multirow[t]{10}{*}{ Loci-Chr14-1 } & $q F E-C h r 14-1$ & 2018 & Bin1494 & Bin1495 & 3.65 & -0.01 & 7.66 & & & & & & \\
\hline & $q F E-C h r 14-2$ & 2018 & Bin 1508 & Bin1509 & 4.60 & -0.01 & 9.54 & & & & & & \\
\hline & & 2018 & Bin1516 & Bin 1517 & 3.17 & -0.01 & 6.68 & & & & & & \\
\hline & qFS-Chr14-1 & 2016 & Bin1501 & Bin 1502 & & & & 3.08 & 0.30 & 5.42 & & & \\
\hline & $q F U-C h r 14-1$ & 2018 & $\operatorname{Bin} 1520$ & $\operatorname{Bin} 1521$ & & & & 3.52 & 0.26 & 8.16 & & & \\
\hline & $q F M-C h r 14-1$ & 2017 & Bin 1502 & Bin 1503 & 3.11 & -0.07 & 4.59 & & & & & & \\
\hline & $q F M-C h r 14-2$ & 2016 & Bin 1521 & Bin 1522 & & & & & & & 2.54 & -0.10 & 5.57 \\
\hline & & 2016 & Bin 1528 & Bin1529 & & & & & & & 2.37 & -0.09 & 4.99 \\
\hline & $q F L-C h r 14-2$ & 2018 & Bin 1517 & Bin 1518 & & & & 2.13 & -0.23 & 4.01 & & & \\
\hline & $q F L-C h r 14-3$ & 2016 & Bin 1529 & Bin 1530 & & & & & & & 2.13 & 0.21 & 4.40 \\
\hline \multirow[t]{2}{*}{ Loci-Chr14-2 } & $q F M-C h r 14-4$ & 2016 & $\operatorname{Bin} 1550$ & Bin1551 & 4.63 & -0.12 & 8.82 & & & & & & \\
\hline & $q F E-C h r 14-3$ & 2018 & Bin 1553 & Bin 1554 & & & & 2.25 & 0.01 & 4.60 & & & \\
\hline \multirow[t]{12}{*}{ Loci-Chr15-1 } & $q F M-C h r 15-1 *$ & 2016 & Bin1568 & Bin 1569 & & & & 2.08 & -0.08 & 4.11 & & & \\
\hline & & 2018 & Bin1571 & Bin 1572 & & & & & & & 3.55 & -0.06 & 7.37 \\
\hline & & 2016 & Bin 1579 & $\operatorname{Bin} 1580$ & & & & 3.84 & -0.11 & 7.42 & & & \\
\hline & & 2016 & Bin 1580 & Bin 1581 & & & & 3.38 & 0.11 & 7.22 & & & \\
\hline & & 2016 & Bin 1589 & Bin 1590 & & & & 2.48 & -0.09 & 4.88 & 2.42 & 0.10 & 6.45 \\
\hline & & 2018 & Bin1594 & Bin 1595 & & & & & & & 4.94 & 0.07 & 10.49 \\
\hline & $q F L-C h r 15-1$ & 2017 & Bin 1599 & Bin 1600 & & & & & & & 2.47 & -0.23 & 5.15 \\
\hline & $q F U-C h r 15-1$ & 2016 & Bin 1579 & Bin 1580 & & & & 2.97 & -0.18 & 5.70 & & & \\
\hline & $q F U-C h r 15-2$ & 2016 & Bin 1599 & Bin 1600 & & & & & & & 2.65 & 0.23 & 5.54 \\
\hline & $q F E-C h r 15-1$ & 2016 & Bin 1589 & Bin 1590 & 2.04 & 0.02 & 4.52 & & & & & & \\
\hline & & 2016 & Bin 1596 & Bin 1597 & & & & & & & 2.80 & 0.02 & 5.68 \\
\hline & & 2016 & Bin 1599 & Bin 1600 & 2.08 & 0.02 & 4.22 & & & & & & \\
\hline \multirow[t]{2}{*}{ Loci-Chr15-2 } & $q F S-C h r 15-1$ & 2017 & Bin 1659 & Bin 1660 & & & & 2.14 & 0.29 & 3.73 & & & \\
\hline & qFM-Chr15-2 & 2017 & Bin1678 & Bin1679 & & & & 2.02 & -0.05 & 2.84 & & & \\
\hline
\end{tabular}


Table 6 (continued)

\begin{tabular}{|c|c|c|c|c|c|c|c|c|c|c|c|c|c|}
\hline \multirow[t]{2}{*}{ Cluster } & \multirow[t]{2}{*}{ QTL } & \multirow[t]{2}{*}{ Year } & \multicolumn{2}{|c|}{ Flanking markers } & \multicolumn{3}{|c|}{ Under $\mathrm{E} 1^{\mathrm{c}}$} & \multicolumn{3}{|c|}{ Under E2 } & \multicolumn{3}{|c|}{ Mapping by $D$-value } \\
\hline & & & & & LOD & Additive & $\operatorname{Var} \%{ }^{\mathrm{b}}$ & LOD & Additive & Var\% & LOD & Additive & Var\% \\
\hline \multirow[t]{5}{*}{ Loci-Chr15-3 } & $q F M-C h r 15-3$ & 2017 & Bin1689 & Bin1690 & 5.53 & -0.10 & 9.13 & & & & & & \\
\hline & & 2016 & Bin 1690 & Bin1691 & & & & 3.48 & -0.10 & 6.72 & & & \\
\hline & & 2017 & Bin 1690 & Bin1691 & & & & 2.48 & -0.06 & 3.84 & & & \\
\hline & $q F L-C h r 15-2$ & 2017 & Bin1694 & Bin1695 & & & & 2.31 & 0.22 & 3.71 & & & \\
\hline & & 2018 & Bin1707 & Bin1708 & & & & 2.02 & 0.23 & 4.00 & & & \\
\hline \multirow[t]{2}{*}{ Loci-Chr16-1 } & $q F E-C h r 16-1$ & 2016 & Bin 1718 & Bin1719 & & & & 2.94 & -0.02 & 5.94 & & & \\
\hline & $q F L-C h r 16-1$ & 2016 & $\operatorname{Bin} 1760$ & Bin1761 & & & & 2.13 & -0.21 & 3.26 & & & \\
\hline \multirow[t]{3}{*}{ Loci-Chr16-2 } & $q F L-C h r 16-2$ & 2017 & Bin 1784 & Bin1785 & & & & 2.10 & -0.20 & 3.38 & & & \\
\hline & $q F M-C h r 16-1 *$ & 2016 & Bin 1817 & Bin1818 & & & & & & & 2.03 & -0.09 & 4.38 \\
\hline & & 2016 & Bin 1821 & Bin 1822 & & & & 2.11 & 0.08 & 3.92 & 3.10 & -0.11 & 6.59 \\
\hline \multirow[t]{2}{*}{ Loci-Chr18-1 } & $q F U-C h r 18-1$ & 2016 & Bin 1904 & Bin1905 & 2.45 & 0.20 & 5.13 & & & & & & \\
\hline & $q F S-C h r l 8-1$ & 2017 & Bin1909 & Bin1910 & & & & & & & 3.33 & 0.39 & 6.51 \\
\hline \multirow[t]{3}{*}{ Loci-Chr19-1 } & $q F U-C h r 19-1$ & 2017 & Bin2057 & Bin2058 & 2.28 & -0.17 & 4.97 & & & & & & \\
\hline & $q F M-C h r 19-1$ & 2017 & Bin2061 & Bin2062 & & & & & & & 3.00 & -0.05 & 6.15 \\
\hline & qFS-Chr19-1 & 2017 & Bin2066 & Bin2067 & & & & & & & 2.75 & 0.36 & 5.35 \\
\hline \multirow[t]{4}{*}{ Loci-Chr21-1 } & $q F M-C h r 21-1$ & 2017 & $\operatorname{Bin} 2279$ & $\operatorname{Bin} 2280$ & & & & 2.05 & -0.05 & 2.71 & & & \\
\hline & & 2016 & $\operatorname{Bin} 2285$ & Bin2286 & 2.00 & 0.08 & 3.77 & & & & & & \\
\hline & & 2016 & $\operatorname{Bin} 2292$ & Bin 2293 & 2.77 & 0.09 & 5.15 & & & & & & \\
\hline & $q F E-C h r 21-1$ & 2018 & $\operatorname{Bin} 2279$ & $\operatorname{Bin} 2280$ & & & & & & & 4.21 & -0.02 & 8.83 \\
\hline \multirow[t]{6}{*}{ Loci-Chr21-2 } & $q F M-C h r 21-2$ & 2017 & $\operatorname{Bin} 2330$ & $\operatorname{Bin} 2331$ & & & & & & & 2.75 & 0.06 & 5.77 \\
\hline & $q F E-C h r 21-2$ & 2017 & $\operatorname{Bin} 2336$ & $\operatorname{Bin} 2337$ & & & & 2.56 & 0.02 & 4.76 & & & \\
\hline & $q F E-C h r 21-3$ & 2017 & $\operatorname{Bin} 2345$ & Bin2346 & 2.28 & 0.02 & 4.37 & & & & & & \\
\hline & $q F L-C h r 21-1$ & 2017 & $\operatorname{Bin} 2335$ & Bin2336 & & & & 2.84 & 0.24 & 4.87 & & & \\
\hline & $q F L-C h r 21-2$ & 2016 & $\operatorname{Bin} 2338$ & Bin2339 & & & & 3.19 & 0.28 & 5.65 & & & \\
\hline & & 2016 & $\operatorname{Bin} 2347$ & $\operatorname{Bin} 2348$ & & & & 2.25 & 0.24 & 4.10 & & & \\
\hline \multirow[t]{2}{*}{ Loci-Chr22-1 } & $q F S-C h r 22-1$ & 2018 & $\operatorname{Bin} 2411$ & $\operatorname{Bin} 2412$ & & & & & & & 2.72 & 0.52 & 6.64 \\
\hline & $q F E-C h r 22-2$ & 2017 & $\operatorname{Bin} 2412$ & $\operatorname{Bin} 2413$ & & & & 2.55 & 0.02 & 4.44 & & & \\
\hline \multirow[t]{5}{*}{ Loci-Chr23-1 } & $q F L-C h r 23-1$ & 2018 & Bin2499 & $\operatorname{Bin} 2500$ & & & & & & & 3.22 & 0.22 & 6.72 \\
\hline & & 2018 & $\operatorname{Bin} 2503$ & Bin2504 & 2.11 & 0.24 & 4.73 & & & & & & \\
\hline & $q F L-C h r 23-2$ & 2018 & $\operatorname{Bin} 2521$ & Bin2522 & 2.83 & -0.33 & 5.93 & & & & & & \\
\hline & $q F M-C h r 23-1$ & 2017 & $\operatorname{Bin} 2537$ & $\operatorname{Bin} 2538$ & & & & 2.06 & 0.05 & 2.73 & & & \\
\hline & $q F U-C h r 23-1$ & 2017 & $\operatorname{Bin} 2554$ & $\operatorname{Bin} 2555$ & & & & & & & 2.05 & 0.21 & 4.38 \\
\hline \multirow[t]{2}{*}{ Loci-Chr24-1 } & $q F U-C h r 24-1$ & 2016 & $\operatorname{Bin} 2558$ & Bin2559 & & & & & & & 3.37 & -0.30 & 6.93 \\
\hline & $q F L-C h r 24-1$ & 2017 & $\operatorname{Bin} 2558$ & Bin2559 & 2.67 & 0.26 & 4.74 & & & & & & \\
\hline \multirow[t]{13}{*}{ Loci-Chr24-2 } & $q F S-C h r 24-1$ & 2017 & $\operatorname{Bin} 2578$ & Bin 2579 & 6.25 & 0.56 & 11.19 & & & & & & \\
\hline & & 2016 & $\operatorname{Bin} 2584$ & Bin2585 & 4.06 & 0.35 & 7.94 & & & & & & \\
\hline & & 2017 & $\operatorname{Bin} 2579$ & $\operatorname{Bin} 2580$ & & & & 2.90 & 0.35 & 5.39 & & & \\
\hline & & 2016 & $\operatorname{Bin} 2581$ & $\operatorname{Bin} 2582$ & & & & 2.60 & 0.27 & 4.66 & & & \\
\hline & $q F S-C h r 24-2$ & 2017 & $\operatorname{Bin} 2592$ & $\operatorname{Bin} 2593$ & 7.19 & 0.60 & 12.73 & & & & & & \\
\hline & & 2016 & $\operatorname{Bin} 2593$ & Bin2594 & 6.83 & 0.44 & 12.90 & & & & & & \\
\hline & & 2017 & $\operatorname{Bin} 2596$ & Bin 2597 & & & & 2.74 & 0.34 & 5.10 & & & \\
\hline & $q F E-C h r 24-1$ & 2018 & $\operatorname{Bin} 2584$ & $\operatorname{Bin} 2585$ & 3.70 & 0.01 & 7.59 & & & & & & \\
\hline & $q F U-C h r 24-2$ & 2016 & $\operatorname{Bin} 2586$ & Bin2587 & & & & & & & 2.23 & 0.24 & 4.52 \\
\hline & $q F L-C h r 24-2$ & 2016 & $\operatorname{Bin} 2581$ & Bin2582 & & & & 2.66 & 0.25 & 4.52 & & & \\
\hline & & 2016 & $\operatorname{Bin} 2587$ & $\operatorname{Bin} 2588$ & 2.61 & 0.26 & 4.67 & & & & & & \\
\hline & & 2017 & $\operatorname{Bin} 2587$ & Bin2588 & & & & 3.27 & 0.26 & 5.68 & & & \\
\hline & $q F L-C h r 24-3$ & 2016 & $\operatorname{Bin} 2593$ & Bin2594 & & & & 4.08 & 0.31 & 6.80 & & & \\
\hline
\end{tabular}


Table 6 (continued)

\begin{tabular}{|c|c|c|c|c|c|c|c|c|c|c|c|c|c|}
\hline \multirow[t]{2}{*}{ Cluster } & \multirow[t]{2}{*}{ QTL } & \multirow[t]{2}{*}{ Year } & \multicolumn{2}{|c|}{ Flanking markers } & \multicolumn{3}{|c|}{ Under $\mathrm{E} 1^{\mathrm{c}}$} & \multicolumn{3}{|c|}{ Under E2 } & \multicolumn{3}{|c|}{ Mapping by $D$-value } \\
\hline & & & & & LOD & Additive & $\operatorname{Var} \%^{\mathrm{b}}$ & LOD & Additive & $\operatorname{Var} \%$ & LOD & Additive & Var\% \\
\hline \multirow[t]{2}{*}{ Loci-Chr24-3 } & $q F S-C h r 24-3$ & 2017 & Bin2601 & Bin 2602 & 4.78 & 0.51 & 8.72 & & & & & & \\
\hline & $q F L-C h r 24-4^{*}$ & 2016 & Bin2606 & Bin 2607 & 2.27 & 0.25 & 4.09 & 2.13 & 0.23 & 3.64 & & & \\
\hline \multirow[t]{2}{*}{ Loci-Chr24-4 } & $q F L-C h r 24-5$ & 2017 & $\operatorname{Bin} 2627$ & $\operatorname{Bin} 2628$ & & & & & & & 2.10 & 0.22 & 4.47 \\
\hline & $q F U-C h r 24-3$ & 2017 & $\operatorname{Bin} 2630$ & $\operatorname{Bin} 2631$ & 2.74 & 0.19 & 5.78 & & & & & & \\
\hline \multirow[t]{3}{*}{ Loci-Chr25-1 } & $q F L-C h r 25-1$ & 2017 & $\operatorname{Bin} 2674$ & $\operatorname{Bin} 2675$ & & & & & & & 2.07 & -0.21 & 4.35 \\
\hline & $q F U-C h r 25-1$ & 2016 & $\operatorname{Bin} 2681$ & $\operatorname{Bin} 2682$ & 2.20 & 0.18 & 4.54 & & & & & & \\
\hline & $q F L-C h r 25-2$ & 2017 & Bin 2683 & Bin2684 & & & & & & & 4.62 & -0.31 & 10.09 \\
\hline \multirow[t]{5}{*}{ Loci-Chr25-2 } & $q F E-C h r 25-1$ & 2017 & $\operatorname{Bin} 2695$ & $\operatorname{Bin} 2696$ & & & & & & & 2.90 & -0.02 & 5.95 \\
\hline & $q F M-C h r 25-1 *$ & 2016 & $\operatorname{Bin} 2700$ & $\operatorname{Bin} 2701$ & 2.48 & 0.09 & 4.91 & & & & 2.06 & 0.08 & 4.32 \\
\hline & & 2016 & Bin2706 & $\operatorname{Bin} 2707$ & 2.47 & 0.08 & 4.56 & & & & & & \\
\hline & $q F S-C h r 25-1$ & 2016 & $\operatorname{Bin} 2707$ & $\operatorname{Bin} 2708$ & 2.03 & -0.24 & 3.66 & & & & & & \\
\hline & & 2016 & $\operatorname{Bin} 2712$ & $\operatorname{Bin} 2713$ & 2.67 & -0.28 & 4.78 & & & & & & \\
\hline \multirow[t]{3}{*}{ Loci-Chr25-3 } & $q F S-C h r 25-2 *$ & 2016 & Bin 2769 & $\operatorname{Bin} 2770$ & 3.21 & 0.30 & 5.79 & & & & 2.17 & 0.27 & 4.95 \\
\hline & $q F U-C h r 25-2$ & 2018 & $\operatorname{Bin} 2763$ & $\operatorname{Bin} 2764$ & & & & & & & 2.70 & -0.31 & 5.72 \\
\hline & & 2018 & Bin 2774 & Bin 2775 & & & & & & & 2.76 & -0.32 & 5.85 \\
\hline \multirow[t]{2}{*}{ Loci-Chr26-1 } & $q F L-C h r 26-1$ & 2016 & Bin 2808 & $\operatorname{Bin} 2809$ & 2.09 & 0.23 & 3.65 & & & & & & \\
\hline & $q F M-C h r 26-1$ & 2016 & $\operatorname{Bin} 2810$ & $\operatorname{Bin} 2811$ & & & & 2.37 & -0.08 & 4.52 & & & \\
\hline
\end{tabular}

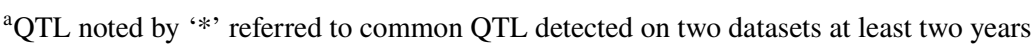

${ }^{b}$ Phenotypic variation explained by a single locus QTL

${ }^{\mathrm{c}} \mathrm{E} 1$, salt stress condition; E2, normal condition; $D$-value, the difference values between salt stress and normal conditions

\section{Genes within QTL for FL conferring fatty acid synthesis and degradation}

Among the 40 QTL identified for FL, 9 were detectable in $D$-value, 13 positive and 18 negative effect QTL among the remaining 31 QTL were observed on E1 or E2, respectively. This examination failed to find the corresponding physical location of some SSR markers on the genome, 10 of which were QTL with positive effect and 11 QTL with negative effect for FL were subjected to gene functional prediction. The physical locations of these QTL on the reference genome of $G$. hirsutum were found, and the numbers of genes in the corresponding regions were 977 and 791 , respectively (Table S7).

We annotated these genes by GO and classified the annotated GO terms. Among them, genes in the QTL region of positive and negative effects were divided into 52 subgroups, belonging to three major categories, CC (cellular components), MF (molecular function), and BP (biological processes) (Fig. S2). There was no significant difference between genes with positive and negative effect QTL in GO classification. GO enrichment of these two kinds of genes showed that the positive effect genes were mainly enriched in three GO items: transmembrane receptor protein tyrosine kinase activity in BP, cell periphery in $\mathrm{CC}$, and system development in MF. The negative effect genes were mainly enriched in abscisic acid glucosyltransferase of BP, membrane-bounded organelle in $\mathrm{CC}$ and cellular protein modification processes in MF (Fig. S3).

To better understand the biological function of candidate genes and their metabolic pathways, all genes $(70,478)$ in upland cotton (Zhang et al. 2015) serve as background and the candidate genes in positive and negative effect QTL were annotated and enriched by KEGG (Fig. 3 and Fig. S4). KEGG annotation showed that the genes in positive QTL were mainly concentrated in three metabolic pathways: plant hormone signal transduction, carbon metabolism and ribosomal, while the genes in negative effect QTL were mainly enriched in plant hormone signal transduction, carbon metabolism, plant pathogen interaction, ribosomes and endocytosis. Interestingly, we found that genes with positive and negative effect QTL are involved in fatty acid metabolism pathways, in which positive effect genes participate in ko00061 (Fatty acid biosynthesis), ko00062 (Fatty acid elongation) and ko01040 (Biosynthesis of unsaturated fatty acids); negative effect genes participate in ko00071 (Fatty acid degradation) (Fig. 4). There are two genes (Gh_ D08G1373 and Gh_D08G1533) involved in fatty acid elongation pathway six genes involved in fatty acid biosynthesis pathway and two genes involved in fatty acid degradation pathway (Table S8). 
Table 7 Putative candidate genes identified within important QTL genomic regions

\begin{tabular}{llll}
\hline QTL & Gene ID & Gene name & Description \\
\hline$q$ FL-Chr01-1 & Gh_A01G0604 & TOR1 & Microtubule-associated protein TORTIFOLIA1 \\
& Gh_A01G0605 & POLIA & DNA polymerase I A, chloroplastic/mitochondrial \\
& Gh_A01G0606 & UGT73C5 & UDP-glycosyltransferase 73C5 \\
& Gh_A01G0607 & PBS1 & Serine/threonine-protein kinase PBS1 \\
& Gh_A01G0608 & NA & NA \\
& Gh_A01G0609 & NA & NA \\
& Gh_A01G0610 & VP22-1 & Vacuolar protein sorting-associated protein 22 homolog 1 \\
& Gh_A01G0611 & FAD4 & Fatty acid desaturase 4, chloroplastic \\
& Gh_A01G0612 & NA & NA \\
& Gh_A01G0613 & CYP716B1 & Cytochrome P450 716B1 \\
Gh_A01G0614 & FAX5 & Protein FATTY ACID EXPORT 5 \\
Gh_A01G0615 & RBP45C & Polyadenylate-binding protein RBP45C \\
GF_-Chr05-5 & Gh_A05G3395 & PPC6-1 & Probable protein phosphatase 2C 33 \\
& Gh_A05G3396 & At1g65240 & Aspartic proteinase-like protein 2 \\
\hline
\end{tabular}

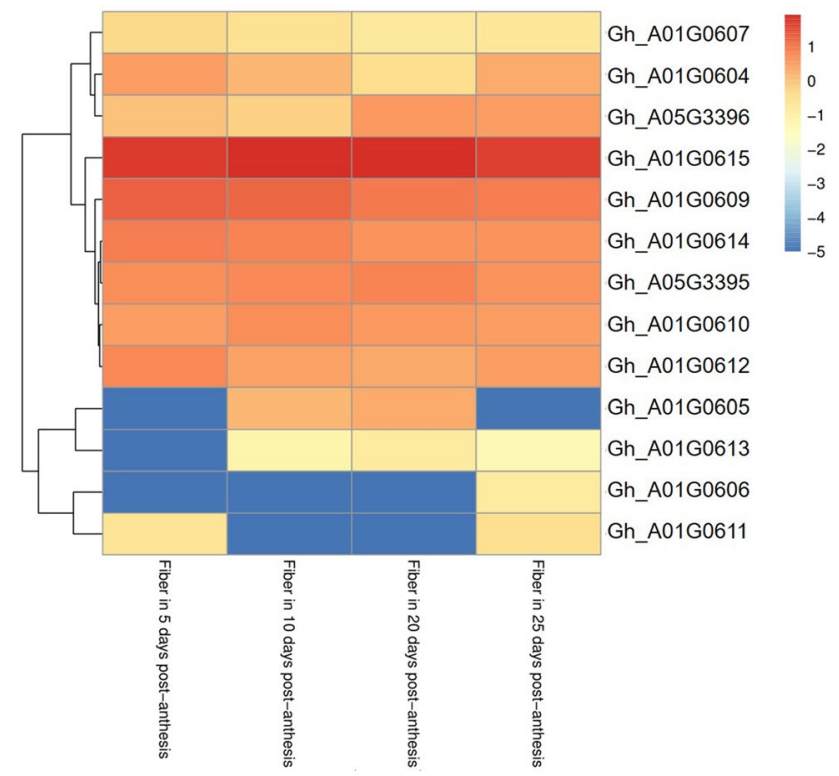

Fig. 1 Expression profiles of candidate genes in fiber development identified within important QTL

\section{Discussion}

\section{The salinity effects on cotton growth and fiber quality traits}

According to the classification standard of soil salt content, in the field trails from 2016 to 2018, the classification of soil salinity is moderate salinization in salt stress condition, and mild salinization in normal condition, as well as the depth of 0-20 cm (Table S1, S2). It can be preliminarily considered that salt treatment is consistent and effective.
By observing the cotton performance in the field, we found that compared with the cotton planted in control soil, the plant height of cotton grown in saline soil was shorter, and leaf margins curled and wilted along the leaf vein. We saw that salt stress negatively affected the vegetative growth of cotton (Fig. S2). The tendency of fiber quality to change among lines in the RIL population under E1 and E2 was different from 2016 to 2018. This could be due to the increase in soil salt content in 2018 and the uneven distribution of salt in the experimental area. It has been reported that low salinity can promote plant growth, but little research has been published to expound the molecular mechanisms of the effects of salt stress on fiber quality in cotton. Salt stress has been shown to result in a considerable decrease in the fresh and dry weights of roots, stems, and leaves (Chartzoulakis and Klapaki 2000; Su et al. 2020). It is reported that in Alhagi pseudoalhagi (a leguminous plant), plant weight increased at low salinity $(50 \mathrm{mM} \mathrm{NaCl})$ but decreased at high salinity (100 and $200 \mathrm{mM} \mathrm{NaCl}$ ) (Kurban H. et al. 1999).

\section{QTL conferring fiber quality under salt stress}

The identification of QTL across multiple environments and populations plays an essential role in marker-assisted selection (MAS) (Jamshed et al. 2016). A total of 318 QTL for FL in upland cotton have been submitted in CottonQTLdb database (https://www.cottonqtldb.org:8081). In the present study, 12 QTL were detected over two years, of which seven, one, four, one and one QTL were detected for FL, FU, FS, $\mathrm{FE}$, and FM. The QTL detected in at least two datasets during the same year were defined as stable QTL. All of 10 stable QTL showed effect values by alleles with different parents. There are two stable QTL underlying FL ( $q F L$ Chrl-1 and $q F L-C h r 24-4)$, one ( $q F S-C h r 25-2)$ controlling 

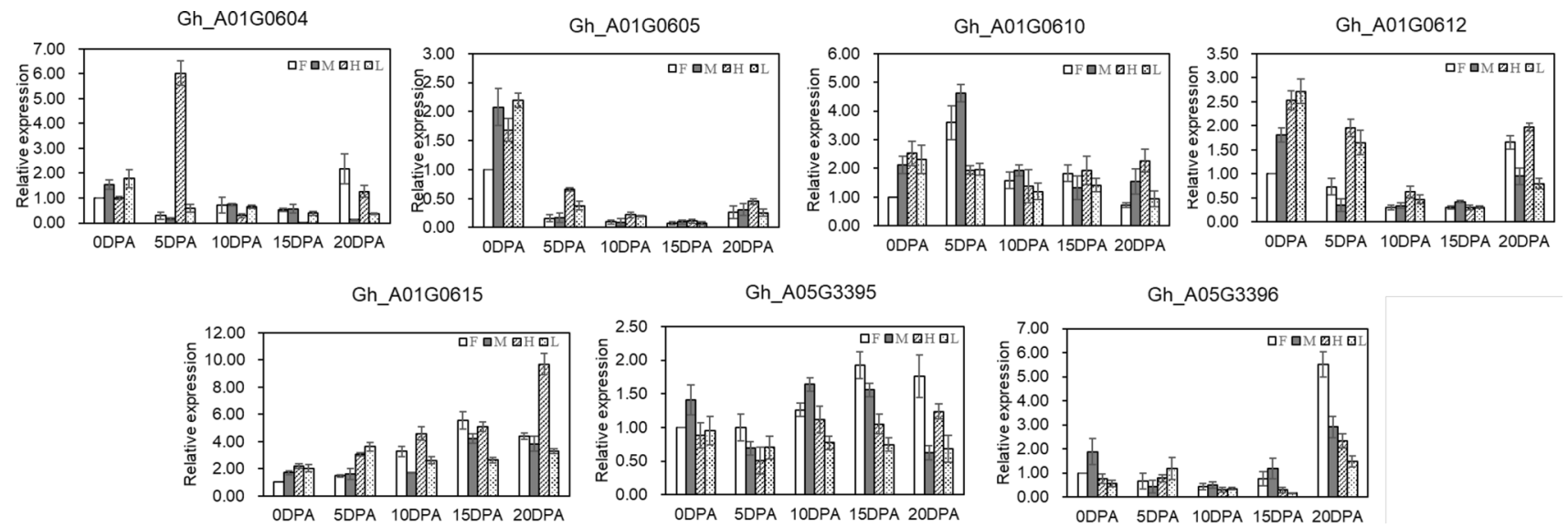

Fig. 2 The relative expression levels of seven candidate genes identified in extreme lines and parents using qRT-PCR. The genes relative expression levels were determined by $2^{-\Delta \Delta C T}$ as expressed and were

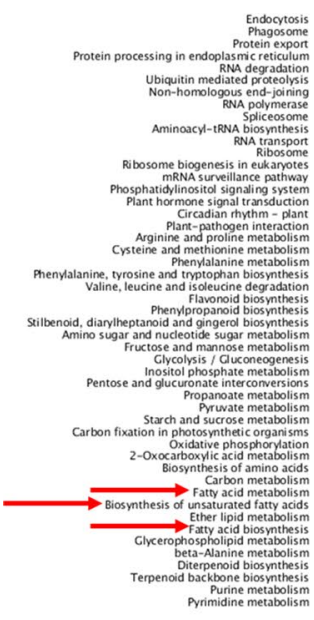

(a)

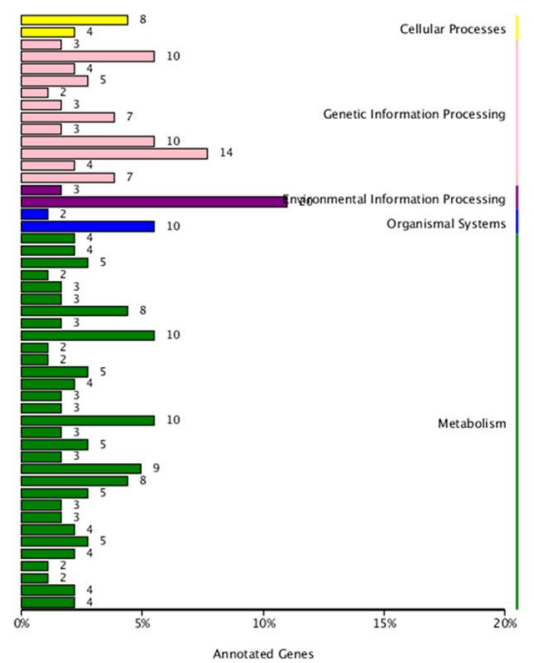

Fig. 3 KEGG functional annotation and classification of genes in positive and negative effect QTL controlling fiber length. a KEGG functional annotation and classification of genes within the posi-

FS and three ( $q F M-C h r 5-2, q F M-C h r 8-2$ and $q F M-C h r 25-1)$ for FM were provided positive provided alleles by the female parent. A total of six QTL were detected in both E1 and E2. Three QTL controlling FL were detected in 2016, of which, $q F L-C h r l-1$ and $q F L-C h r 24-4$ were provided alleles by the female parent and $q F L-C h r 5-5$ was provided alleles positive by the male parent. Two of the QTL that affect FM are located on Chr8 ( $q F M-C h r 8-2, q F M-C h r 8-3)$ and were detected in 2018. One stable QTL $(q F M-C h r 5-2)$ was detected in 2017, where $q F M-C h r 5-2$ and $q F M-C h r 8-2$ demonstrated positive additive effects, and the other QTL, qFM-Chr8-3 showed negative additive effects. The results revealed that these hotspots play important roles in two normalized to the expression level of $G h U B Q 7$ gene. F, GX1135; M, GX100-2; H, extremely long fiber length line; L, extremely short fiber length line
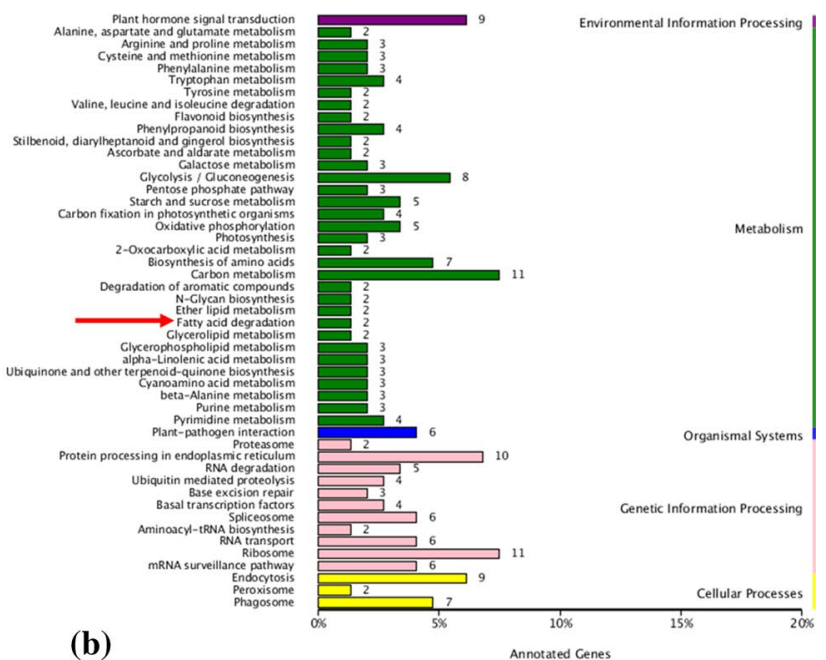

tive effect QTL. b KEGG functional annotation and classification of genes within the negative effect QTL

conditions for diverse fiber quality traits but different mechanisms responded between under E1 and E2.

Additionally, two QTL ( $q F M-C h r 25-1, q F S-C h r 25-2)$ were detected in E1 and $D$-value in 2016. The favorable alleles were provided by the female parent for both FM and FS. Two QTL were detected in E2 and $D$-value in 2016, affecting FM, $q F M$-Chr15-1, the positive allele of which was provided by the male parent and $q F M-C h r 16-1$, the positive allele of which was provided by the female parent. A total of 46 QTL were detected for abiotic and biotic stress resistance in cotton on Chr5 including three hotspots, and 22 QTL on Chr14 including 1 hotspot, and 31 QTL on Chr15 including 2 hotspots (Abdelraheem et al. 2017). The hotspot named 


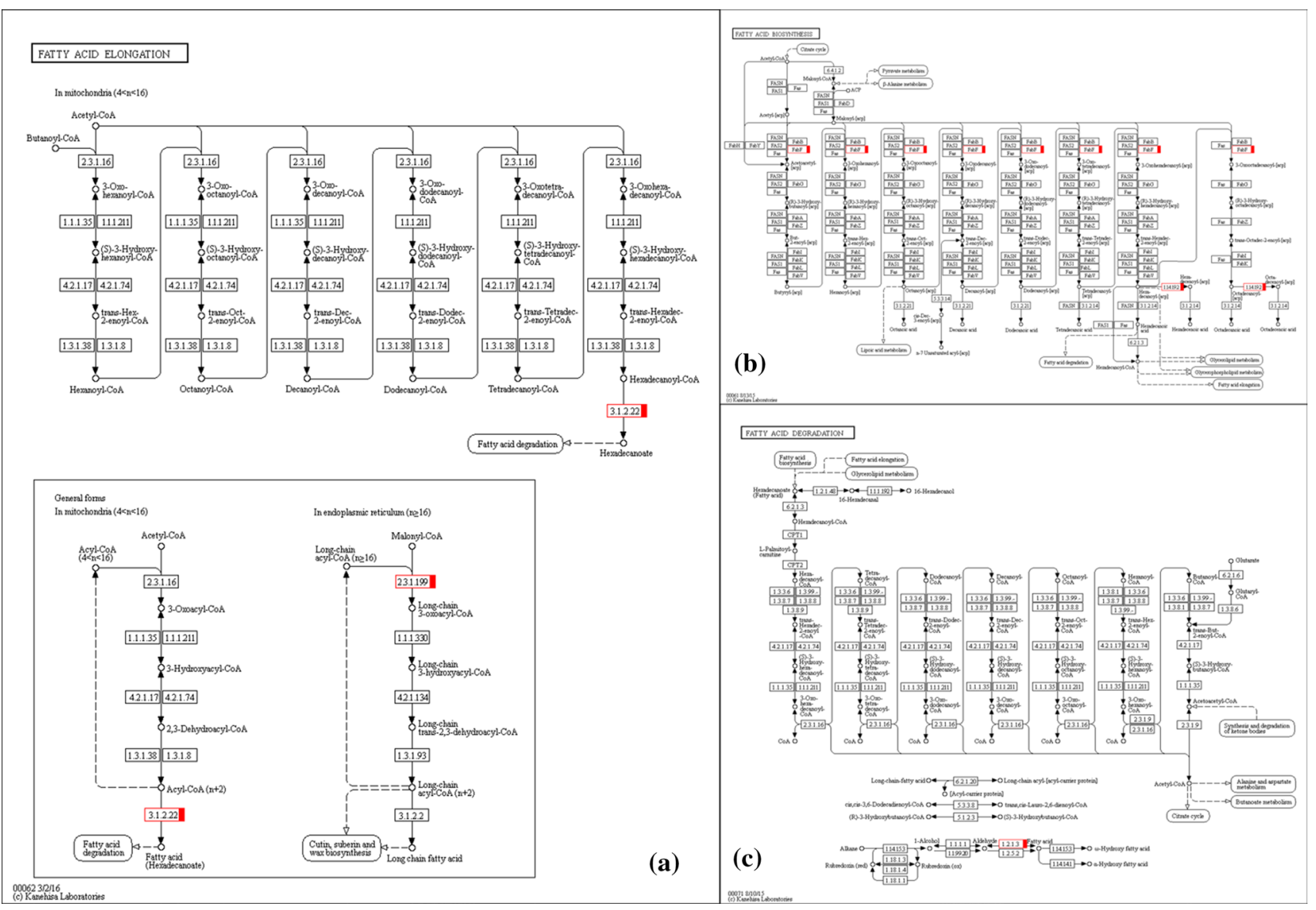

Fig. 4 Genes involved in fatty acid metabolism. a Genes involved in fatty acid elongation pathway. b Genes involved in fatty acid biosynthetic pathway. $\mathbf{c}$ Genes involved in fatty acid degradation pathway

c15-ST-Hotspot-1 was detected for fresh root weight under salt stress condition. The stable QTL, $q F E-C h r 25-1$ in the present study, was detected in $D$-value, consistently with the same chromosome harboring a hotspot for Micronaire under normal irrigation condition (Said et al. 2013) and two hotspots for abiotic and biotic stress resistance underlying Micronaire and fiber elongation in cotton (Abdelraheem et al. 2017). This indicates regions on the same chromosome underlying fiber quality traits showed response to diverse stress conditions. One consistent QTL located on Chr9 and four consistent QTL on Chr15 were detected for shoot height, shoot fresh weight, shoot dry weight, and root dry weight under salt stress in seedling stage (Oluoch et al. 2016).

\section{Stable QTL affecting FL under multiple environments across multiple years}

A QTL cluster is defined as a densely populated QTL region on a chromosome that contains many QTL associated with different traits (Rong et al. 2007). In this study, we identified 35 clusters located on 18 chromosomes. Two stable QTL were located in the cluster, Loci-Chr5-4 controlling FL ( $q F L-C h r 5-5)$ and FM ( $q F M-C h r 5-2)$ under E1 and E2. This cluster harbored six QTL, of which three QTL controlled FS ( $q F S-C h r 5-5, q F S-C h r 5-6, q F S-C h r 5-7)$ and one for FL ( $q F L-C h r 5-5)$ had negative additive effects, while two QTL $q F M-C h r 5-2$ and $q F M-C h r 5-3$ controlled FM showed positive additive effects. Three pleiotropic regions contained two stable QTL ( $q F M$-Chr25-1 and $q F S$ Chr25-2) were detected on Chr25, of which it also controlled $\mathrm{FL}$ and FU simultaneously. This is consistent with previous research that an important cluster with more than three traits, with high broad sense heritability and high percentage of phenotypic variation was identified on Chr25 (Diouf et al. 2018). There were four QTL-clusters on Chr24, six QTL in Loci-Chr24-2, and two QTL in Loci-Chr24-1, Loci-Chr24-3, Loci-Chr24-4, respectively. In Loci-Chr24-2, six QTL had positive additive effects which controlled FL, FS, FE, and FU simultaneously. One stable QTL, $q F L-C h r 24-4$, had positive additive effects that were identified in Loci-Chr24-3 on both E1 and E2, which controlled FS. Naoumkina et al. 
(2019) found 12 genes possessing non-synonymous SNPs (nsSNPs) significantly associated with fiber length on Chr. D11 (Chr24 in present research) using 550 RILs derived from eleven different cultivars. The high correlation of the QTL detected in this study to the previous finding, provides the opportunity for the utilization of these QTL in MAS to improve the fiber quality of upland cotton.

There were six QTL-clusters influencing FL and FS, of which two clusters (Loci-Chr5-1, Loci-Chr5-4) located on Chr5, two (Loci-Chr24-2, Loci-Chr24-1) located on Chr24, one (Loci-Chr7-1) located on Chr7, and one (Loci-Chr14-1) located on Chr14, respectively. Six clusters affecting FL and FS simultaneously received favorable alleles contributed by the same parent under E1 and E2 except Loci-Chr14-1 (Table 6). The cluster located on Chr14 indicated that the correlation between FS and FL was not altered by salt stress.

\section{Advantage of multiple datasets for QTL mapping under salt stress}

In general, when mapping with composite traits, the QTL detected by both constituent and composite traits have a positive correlation in detecting efficacy. Constituent traits can accumulate the genetic effect; however, the efficiency of composite traits is generally high with multiplier effects (Hua et al. 2002). In recent years, composite traits in stress response have been studied using QTL mapping strategies. In a maize example, anthesis silking interval (ASI) index was used to identify the phenotypic value for drought tolerance (Ribaut et al. 1996; Messmer et al. 2009). ASI in tropical open-pollinated varieties was negatively correlated with yields under drought stress, so ASI was one of the most important composite traits in the identified drought tolerance. The phenotypic values of ASI came from the difference between MFLW (male flowering) and FFLW (female flowering). Ribaut et al. (1996) used 142 molecular markers to analyze the characters of MFLW, FFLW, and ASI of a maize $\mathrm{F}_{2}$ population under good irrigation and water stress conditions, respectively. In the present study, the $D$-value was used to represent the effect of salt stress on the RIL population, which was developed as a compound trait. The results showed that 46 QTL were detected in $D$-value, of which 11 could be detected in the constituent traits explaining from 4.32 to $11.19 \%$ of $\mathrm{PV}$ and LOD values ranged 2.06-5.28. Of the 11 QTL, four QTL ( $q F S-C h r 25-2, q F M-$ Chr15-1, qFM-Chr16-1, qFM-Chr25-1) were detected using constituent trait and composite trait in the same year (E1 and $D$-value, E2 and $D$-value), which explained $\mathrm{PV}$, ranging from $4.32 \%$ to $6.59 \%$ (Table 5). Among them, two QTL ( $q F M$ Chr15-1, qFM-Chr16-1) were detected on E2 and D-value, and two ( $q F M$-Chr25-1, qFS-Chr25-2) were detected on E1 and $D$-value. This research exploits and provides an example for QTL effects detected in both the composite trait and the constituent traits for salt-tolerant QTL mapping.

\section{Candidate genes positively regulate fiber elongation}

Fiber length is one of the most important and highly heritable fiber quality trait, which has been successfully used for genetic analysis in cotton and is directly related to its spinning quality. Fiber development is a complex physiological and biochemical process, which goes through multiple stages and involves a multi-level and multi-pathway molecular regulation network. Among them, plant hormones, turgor regulation, and cytoskeleton can all participate in the regulation of cotton fiber growth and development (Ascencio-Ibáñez et al. 2008; Gardiner et al. 2011). Two genes, Gh_A05G3395 and $G h \_A 05 G 3396$, located within $q F L-C h r 5-5$ had homologous genes in Arabidopsis thaliana. Gh_A05G3396 was a membrane-anchored aspartic protease, which contributed to pollen and ovule development. And Gh_A05G3395 is an $\mathrm{Mn}^{2+}$ or $\mathrm{Mg}^{2+}$-dependent protein serine/threonine phosphatase, which may constitute positive regulators in ABAmediated signaling pathways (Vanholme et al. 2014; Xue et al. 2008). Li et al. (2002) cloned a gene (GhTUB1), which encoded $\beta$ subunit of micro-tubulin and predominantly expressed in cotton fiber. He et al. (2008) identified 795 tubulin ESTs (expressed sequence tags), in upland cotton, of which $19 \beta$-TUB genes were cloned. The microtubuleassociated protein encoding gene (Gh_A01G0604) was significant highly expressed in extremely long fiber length line at the earliest time of fiber development (5 DPA). $G h_{-}$ A01G0605 (DNA polymerase I A), Gh_A01G0610 (vacuolar protein sorting-associated protein), $G h \_A 01 G 0612$ (no functional annotation), Gh_A01G0615 (Polyadenylate-binding protein) in extremely long fiber line was significantly higher than that of extremely short fiber line at 10 DPA to 20 DPA. These candidate genes annotated as being associated with plant turgor regulation, cytoskeleton processes, DNA replication, and protein synthesis might positively regulate fiber elongation in A. thaliana or in G. hirsutum, and these two QTL may contribute to fiber quality breeding.

\section{Fiber cell elongation aroused fatty acid synthesis pathway under salt stress}

In total, 977 genes in 10 positive effect QTL and 791 genes in 10 negative effect QTL were used to analyze gene function and the biological processes (Table S7). Interestingly, we found that genes with positive effects participate in ko00061 (Fatty acid biosynthesis), ko00062 (Fatty acid elongation), and ko01040 (Biosynthesis of unsaturated fatty acids); while negative effect genes involved in ko00071 
(Fatty acid degradation). Of these, a gene encoding 3-ketoacyl-CoA synthase 1 belonging to GhKCSI (Gh_D08G1373) was involved in fatty acid elongation pathway (Fig. 3 and 4, Table S8). The biosynthesis of VLCFAs and their transport are required for fiber development (Hu et al. 2007). The heterologous gene KCS of cotton expressed in Arabidopsis thaliana had promoted the elongation of stem cells (Qin et al. 2007; Shi et al. 2006). Furthermore, increased biosynthesis of fatty acid during the fiber cell elongation period implicated that fatty acid was serve as precursors of cutin and cuticular wax in the process (Hu et al. 2019a, b; Fig. 4). We predict that QTL with positive effects on fiber length may increase fiber length by promoting the synthesis and elongation of fatty acids, while QTL with negative effects may be responsible for fatty acid degradation and thus inhibit elongation of fiber cells. Our research provides a foundation for future examination of the role of fatty acid in cotton fiber development under salt stress. However, the regulative mechanism of these genes in fiber elongation still needs to be further verified.

Acknowledgements This research was supported by the National Natural Science Foundation of China (No. 31530053) and National Key R \& D Program for Crop Breeding (2016YFD0101305) to Jinping Hua. We thank Dr. Lingling Ma and Mr. Wei Liang for their contributions on field experimental work.

Author contribution AHG performed two years' experiments, all data analysis and prepared the manuscript. YS performed one-year experiment and data collection. BI and HSN attended bench work and discussion. YH attended discussion and revised the manuscript. YMW maintained experimental platform and performed bench work. JPH conceived the experiments, provided experimental platform and revised the manuscript. All authors approved of the final manuscript.

Data availability All data generated or analyzed in this study included in published article and additional files. All of our raw data are available as Supporting Information: Supplementary tables S3-S5.

\section{Compliance with ethical standards}

Conflict of interest The authors declare that they have no conflict of interest.

Open Access This article is licensed under a Creative Commons Attribution 4.0 International License, which permits use, sharing, adaptation, distribution and reproduction in any medium or format, as long as you give appropriate credit to the original author(s) and the source, provide a link to the Creative Commons licence, and indicate if changes were made. The images or other third party material in this article are included in the article's Creative Commons licence, unless indicated otherwise in a credit line to the material. If material is not included in the article's Creative Commons licence and your intended use is not permitted by statutory regulation or exceeds the permitted use, you will need to obtain permission directly from the copyright holder. To view a copy of this licence, visit http://creativecommons.org/licenses/by/4.0/.

\section{References}

Abdelraheem A, Liu F, Song M, Zhang JF (2017) A meta-analysis of quantitative trait loci for abiotic and biotic stress resistance in tetraploid cotton. Mol Genet Genom 292:1221-1235

Ascencio-Ibáñez JT, Sozzani R, Lee T, Chu T, Wolfinger RD, Cella R, Hanley-Bowdoin L (2008) Global analysis of Arabidopsis gene expression uncovers a complex array of changes impacting pathogen response and cell cycle during geminivirus infection. Plant Physiol 148:436-454

Cai C, Wu S, Niu E, Cheng C, Guo W (2017) Identification of genes related to salt stress tolerance using intron-length polymorphic markers, association mapping and virus-induced gene silencing in cotton. Sci Rep 7:528

Chartzoulakis K, Klapaki G (2000) Response of two greenhouse pepper hybrids to $\mathrm{NaCl}$ salinity during different growth stages. Sci Hortic 86:247-260

Chaudhary B, Hovav R, Rapp R, Verma N, Udall JA, Wendel JF (2008) Global analysis of gene expression in cotton fibers from wild and domesticated Gossypiumbarbadense. Evol Dev 10:567-582

Chaudhary B, Hovav R, Flagel L, Mittler R, Wendel JF (2009) Parallel expression evolution of oxidative stress-related genes in fiber from wild and domesticated diploid and polyploid cotton (Gossypium). BMC Genom 10:378

Chen ZJ, Sreedasyam A, Ando A, Song Q, De Santiago LM, HulseKemp AM, Ding M, Ye W, Kirkbride RC, Jenkins J, Plott C, Lovell J, Lin Y, Vaughn R, Liu B, Simpson S, Scheffler BE, Wen L, Saski CA, Grover CE, Hu G, Conover JL, Carlson JW, Shu S, Boston LB, Williams M, Peterson DG, McGee K, Jones DC, Wendel JF, Stelly DM, Grimwood J, Schmutz J (2020) Genomic diversifications of five Gossypium allopolyploid species and their impact on cotton improvement. Nat Genet 52:525-533

Chinnusamy V, Zhu J, Zhu JK (2006) Salt stress signaling and mechanisms of plant salt tolerance. Genet Eng 27:141-177

Diouf L, Pan Z, He SP, Gong WF, Jia YH, Magwanga R, Romy K, Or Rashid H, Kirungu J, Du XM (2017) High-density linkage map construction and mapping of salt-tolerant QTL at seedling stage in upland cotton using genotyping by sequencing (GBS). Int $\mathrm{J}$ Mol Sci 18:2622

Diouf L, Magwanga R, Gong WF, He S, Pan Z, Jia Y, Kirungu J, Du XM (2018) QTL mapping of fiber quality and yield-related traits in an intra-specific upland cotton using genotype by sequencing (GBS). Int J Mol Sci 19:441

Flowers TJ (2004) Improving crop salt tolerance. J Exp Bot 55:307-319

Foolad MR, Chen FQ (1999) RFLP mapping of QTLs conferring salt tolerance during the vegetative stage in tomato. TheorAppl Genet 99:235-243

Gao Y, Li M, Zhang X, Yang Q, Huang B (2019) Up-regulation of lipid metabolism and glycine betaine synthesis are associated with choline-induced salt tolerance in halophytic seashore paspalum. Plant Cell Environ 43:159-173

Gardiner J, Overall R, Marc J (2011) Putative Arabidopsis homologues of metazoan coiled-coil cytoskeletal proteins. Cell Biol Int 35:767-774

Golldack D, Li C, Mohan H, Probst N (2014) Tolerance to drought and salt stress in plants: unraveling the signaling networks. Front Plant Sci 5:151

Guo JY, Shi GY, Guo XY, Zhang L, Xu W, Wang YM, Su Z, Hua JP (2015) Transcriptome analysis reveals that distinct metabolic pathways operate in salt-tolerant and salt-sensitive upland cotton varieties subjected to salinity stress. Plant Sci 238:33-45

He XC, Qin YM, Xu Y, Hu CY, Zhu YX (2008) Molecular cloning, expression profiling, and yeast complementation of 19-tubulin cDNAs from developing cotton ovules. J Exp Bot 59:2687-2695 
Hu WJ, Chen L, Qiu XY, Wei J, Lu HL, Sun GC (2019) AKR2A participates in the regulation of cotton fibre development by modulating biosynthesis of very-long-chain fatty acids. Plant Biotechnol J 18:526-539

Hu Y, Chen J, Fang L, Zhang Z, Ma W, Niu Y, Ju L, Deng J, Zhao T, Lian J, Baruch K, Fang D, Liu X, Ruan Y, Rahman M, Han J, Wang K, Wang Q, Wu H, Mei G, Zang Y, Han Z, Xu C, Shen W, Yang D, Si Z, Dai F, Zou L, Huang F, Bai Y, Zhang Y, Brodt A, Ben-Hamo H, Zhu X, Zhou B, Guan X, Zhu S, Chen X, Zhang T (2019) Gossypium barbadense and Gossypium hirsutum genomes provide insights into the origin and evolution of allotetraploid cotton. Nat Genet 51:739-748

Hua JP, Xing YZ, Xu CG, Sun XL, Yu SB, Zhang QF (2002) Genetic dissection of an elite rice hybrid revealed that heterozygotes are not always advantageous for performance. Genetics 162:1885-1895

Huang S, Spielmeyer W, Lagudah ES, James RA, Platten JD, Dennis ES, Munns R (2006) A sodium transporter (HKT7) is a candidate for Naxl, a gene for salt tolerance in durum wheat. Plant Physiol $14: 1718-1727$

Ijaz B, Zhao N, Kong J, Hua JP (2019) Fiber quality improvement in upland cotton (Gossypium hirsutum L.): quantitative trait loci mapping and marker assisted selection application. Front Plant Sci 10:1585

Ismail AM, Horie T (2017) Genomics, physiology, and molecular breeding approaches for improving salt tolerance. Annu Rev Plant Biol 68:405-434

Jamshed M, Jia F, Gong J, Palanga KK, Shi Y, Li J, Shang H, Liu A, Chen T, Zhang Z, Cai J, Ge Q, Liu Z, Lu Q, Deng X, Tan Y, Or Rashid H, Sarfraz Z, Hassan M, Gong W, Yuan Y (2016) Identification of stable quantitative trait loci (QTLs) for fiber quality traits across multiple environments in Gossypium hirsutum recombinant inbred line population. BMC Genom 17:197

Kim HJ, Triplett BA (2001) Cotton fiber growth in planta and in vitro. Models for plant cell elongation and cell wall biogenesis. Plant Physiol 127:1361-1366

Kosambi DD (1944) The estimation of map distances from recombination values. Ann Eugen 12:172-175

Kumar V, Singh A, Mithra SV, Krishnamurthy SL, Parida SK, Jain S, Tiwari KK, Kumar P, Rao AR, Sharma SK, Khurana JP, Singh NK, Mohapatra T (2015) Genome-wide association mapping of salinity tolerance in rice (Oryza sativa). DNA Res 22:133-145

Kurban H, Saneoka H, Nehira K, Adilla R, Premachandra G, Fujita K (1999) Effect of salinity on growth, photosynthesis and mineral composition in leguminous plant Alhagi pseudoalhagi (Bieb.). Soil Sci Plant Nutr 45:851-862

Lee GJ, Boerma HR, Villagarcia MR, Zhou X, Carter TE Jr, Li Z, Gibbs MO (2004) A major QTL conditioning salt tolerance in S-100 soybean and descendent cultivars. Theor Appl Genet 109:1610-1619

Li X, Cai L, Cheng N, Liu J (2002) Molecular characterization of the cotton GhTUB1 gene that is preferentially expressed in fiber. Plant Physiol 130:666-674

Li H, Handsaker B, Wysoker A, Fennell T, Ruan J, Homer N, Marth G, Abecasis G, Durbin R (2009) The sequence alignment/map format and SAMtools. Bioinformatics 25:2078-2079

Li W, Zhao F, Fang W, Xie D, Hou J, Yang X, Zhao Y, Tang Z, Nie L, Lv S (2015) Identification of early salt stress responsive proteins in seedling roots of upland cotton (Gossypium hirsutum L.) employing iTRAQ-based proteomic technique. Front Plant Sci. https://doi.org/10.3389/fpls.2015.00732

Liang Q, Hu C, Hua H, Li Z, Hua J (2013) Construction of a linkage map and QTL mapping for fiber quality traits in upland cotton (Gossypium hirsutum L.). Chinese Sci Bull 58:3233-3243

Liu A, Xiao Z, Li M, Wong F, Yung W, Ku Y, Wang Q, Wang X, Xie M, Yim AK, Chan T, Lam H (2019) Transcriptomic reprogramming in soybean seedlings under salt stress. Plant Cell Environ 42:98-114

Livak KJ, Schmittgen TD (2001) Analysis of relative gene expression data using real-time quantitative PCR and the $2^{-\Delta \Delta C T}$ method. Methods 25:402-408

Ma LL, Zhao YP, Wang YM, Shang LG, Hua JP (2017) QTLs analysis and validation for fiber quality traits using maternal backcross population in upland cotton. Front Plant Sci 8:2168

Ma LL, Wang YM, Ijaz B, Hua JP (2019) Cumulative and different genetic effects contributed to yield heterosis using maternal and paternal backcross populations in upland cotton. Sci Rep 9:3984

Ma Q, Sun M, Kang H, Lu J, You C, Hao Y (2019) A CIPK protein kinase targets sucrose transporter MdSUT2.2 at Ser254 for phosphorylation to enhance salt tolerance. Plant Cell Environ 42:918-930

Mano Y, Takeda K (1997) Mapping quantitative trait loci for salt tolerance at germination and the seedling stage in barley (Hordeum vulgare L.). Euphytica 94:263-272

McKenna A, Hanna M, Banks E, Sivachenko A, Cibulskis K, Kernytsky A, Garimella K, Altshuler D, Gabriel S, Daly M, DePristo MA (2010) The genome analysis toolkit: a mapreduce framework for analyzing next-generation dna sequencing data. Genome Res 20:1297-1303

Messmer R, Fracheboud Y, Bänziger M, Vargas M, Stamp P, Ribaut J (2009) Drought stress and tropical maize: QTL-by-environment interactions and stability of QTLs across environments for yield components and secondary traits. Theor Appl Genet 119:913-930

NaoumkinaThyssenJenkinsMcCartyFlorane MGNFDDJNCB (2019) Genetic and transcriptomic dissection of the fiber length trait from a cotton (Gossypiumhirsutum L.) MAGIC population. BMC Genomics. https://doi.org/10.1186/s12864-019-5427-5

Oluoch G, Zheng J, Wang X, Khan M, Zhou Z, Cai X, Wang C, Wang Y, Li Y, Wang H, Liu F, Wang K (2016) QTL mapping for salt tolerance at seedling stage in the interspecific cross of Gossypium tomentosum with Gossypium hirsutum. Euphytica 209:223-235

Park W, Scheffler B, Bauer P, Campbell B (2012) Genome-wide identification of differentially expressed genes under water deficit stress in upland cotton (Gossypium hirsutum L.). BMC Plant Biol. https ://doi.org/10.1186/1471-2229-12-90

Paterson AH, Brubaker CL, Wendel JF (1993) A rapid method for extraction of cotton. Plant Mol Biol Rep 11:122-127

Pawłowicz I, Masajada K (2019) Aquaporins as a link between water relations and photosynthetic pathway in abiotic stress tolerance in plants. Gene 687:166-172

Qin YM, Zhu YX (2011) How cotton fibers elongate: a tale of linear cell-growth mode. CurrOpin Plant Biol 14:106-111

Qin YM, Hu CY, Pang Y, Kastaniotis JA, Hiltunen JK, Zhu YX (2007) Saturated very-long-chain fatty acids promote cotton fiber and Arabidopsis cell elongation by activating ethylene biosynthesis. Plant Cell 19:3692-3704

Rhoades J (1996) Salinity: electrical conductivity and total dissolved solids. Chem Methods 142:31-33

Ribaut JM, Hoisington DA, Deutsch JA, Jiang C, Gonzalez-de-Leon D (1996) Identification of quantitative trait loci under drought conditions in tropical maize. 1. flowering parameters and the anthesissilking interval. Theor Appl Genet 92:905-914

Richter JA, Behr JH, Erban A, Kopka J, Zörb C (2019) Ion-dependent metabolic responses of Vicia faba L. to salt stress. Plant Cell Environ 42:295-309

Rong J, Feltus F, Waghmare V, Pierce G, Chee P, Draye X, Saranga Y, Wright R, Wilkins T, May O, Smith C, Gannaway J, Wendel J, Paterson A (2007) Meta-analysis of polyploid cotton QTL shows unequal contributions of subgenomes to a complex network of genes and gene clusters implicated in lint fiber development. Genetics 176:2577-2588 
Said JI, Lin Z, Zhang X, Song M, Zhang J (2013) A comprehensive meta QTL analysis for fiber quality, yield, yield related and morphological traits, drought tolerance, and disease resistance in tetraploid cotton. BMC Genom 14:776

Shang LG, Liang QZ, Wang YM, Wang XC, Wang KB, Abduweli A, Ma LL, Cai SH, Hua JP (2015) Identification of stable QTLs controlling fiber traits properties in multi-environment using recombinant inbred lines in upland cotton (Gossypium hirsutum L.). Euphytica 205:877-888

Shang LG, Liang QZ, Wang YM, Zhao YP, Wang KB, Hua JP (2016) Epistasis together with additive, partial dominance, over-dominance and QTL by environment interactions as the primary genetic basis of yield heterosis in upland cotton. Theor Appl Genet 129:1429-1446

Shang L, Wang Y, Wang X, Liu F, Abduweli A, Cai S, Hua J (2016) Genetic analysis and QTL detection on fiber traits using two recombinant inbred lines and their backcross populations in upland cotton. G3 6(9):2717-2724

Shao Q, Zhang F, Tang S, Liu Y, Fang X, Liu D, Liu D, Zhang J, Teng Z, Paterson AH, Zhang Z (2014) Identifying QTL for fiber quality traits with three upland cotton (Gossypium hirsutum L.) populations. Euphytica 198:43-58

Shi YH, Zhu SW, Mao XZ, Feng JX, Zhu YX (2006) Transcriptome profiling, molecular biological, and physiological studies reveal a major role for ethylene in cotton fiber cell elongation. Plant Cell 18:651-664

Shi GY, Guo XY, Guo YJ, Liu L, Hua JP (2015) Analyzing serial cDNA libraries revealed reactive oxygen species and gibberellins signaling pathways in the salt response of upland cotton (Gossypium hirsutum L.). Plant Cell Rep 34:1005-1023

Shinozawa A, Otake R, Takezawa D, Umezawa T, Komatsu K, Tanaka K, Amagai A, Ishikawa S, Hara Y, Kamisugi Y, Cuming AC, Hori K, Ohta H, Takahashi F, Shinozaki K, Hayashi T, Taji T, Sakata Y (2019) SnRK2 protein kinases represent an ancient system in plants for adaptation to a terrestrial environment. Commun Biol 2:1-13

Su Y, Liang W, Liu JZ, Wang YM, Zhao YP, Babar I, Hua JP (2017a) Overexpression of GhDofl improved salt and cold tolerance and seed oil content in Gossypium hirsutum. J Plant Physiol 218:222-234

Su Y, Wang YM, Zhen J, Zhang X, Chen ZW, Li L, Huang Y, Hua JP (2017b) SnRK2 homologs in Gossypium and GhSnRK2.6 improved salt tolerance in transgenic upland cotton and Arabidopsis. Plant Mol Biol Rep 35:442-456

Su Y, Guo A, Huang Y, Wang YM, Hua JP (2020) GhCIPK6a increases salt tolerance in transgenic upland cotton by involving in ROS scavenging and MAPK signaling pathways. BMC Plant Biol 20:421

Sun Z, Li H, Zhang Y, Li Z, Ke H, Wu L, Zhang G, Wang X, Ma Z (2018) Identification of SNPs and candidate genes associated with salt tolerance at the seedling stage in cotton (Gossypium hirsutum L.). Front Plant Sci. https://doi.org/10.3389/fpls.2018.01011

Sun H, Meng M, Yan Z, Lin Z, Nie X, Yang X (2019) Genome-wide association mapping of stress-tolerance traits in cotton. Crop J $7: 77-88$
Tounsi S, Ben AS, Masmoudi K, Sentenac H, Brini F, Very AA (2016) Characterization of two HKT1; 4 transporters from Triticum monococcum to elucidate the determinants of the wheat salt tolerance Naxl QTL. Plant Cell Physiol 57:2047-2057

Wang J, Huang R (2019) Modulation of ethylene and ascorbic acid on reactive oxygen species scavenging in plant salt response. Front Plant Sci 10:319

Wang Z, Zhu S, Yu R et al (1993) Chinese saline soil. Science Press, Beijing, pp 333-335

Wang S, Basten C, Zeng Z (2007) Windows QTL cartographer 25. North Carolina State University, Raleigh, NC, Department of Statistics

Wang X, Gao F, Bing J, Sun W, Feng X, Ma X, Zhou Y, Zhang G (2019) Overexpression of the Jojoba aquaporin gene, ScPIP1, enhances drought and salt tolerance in transgenic Arabidopsis. Int J Mol Sci 20:153

Wendel JF, Brubaker CL, Seelanan T (2010) The origin and evolution of Gossypium. Physiology of Cotton Springer, Netherlands

Xie W, Feng Q, Yu H, Huang X, Zhao Q, Xing Y, Yu S, Han B, Zhang Q (2010) Parent-independent genotyping for constructing an ultrahigh-density linkage map based on population sequencing. Proc Natl Acad Sci USA 107:10578-10583

Xue T, Wang D, Zhang S, Ehlting J, Ni F, Jakab S, Zheng C, Zhong Y (2008) Genome-wide and expression analysis of protein phosphatase 2C in rice and Arabidopsis. BMC Genom 9:550

Yamaguchi T, Blumwald E (2005) Developing salt-tolerant crop plants: challenges and opportunities. Trends Plant Sci 10:615-620

Zhang LN, Ye WW, Wang JJ, Fan BX (2010) Study of salinity-tolerance with SSR markers on Gossypium hirsutum. Cotton Sci 22:175-180

Zhang X, Zhen JB, Li Z, Kang D, Yang YM, Kong J, Hua JP (2011) Expression profile of early responsive genes under salt stress in upland cotton (Gossypium hirsutum L.). Plant Mol Biol Rep 29:626-637

Zhang TZ, Hu Y, Jiang W, Fang L, Guan X, Chen J, Zhang J, Saski CA, Scheffler BE, Stelly DM, Hulse-Kemp AM, Wan Q, Liu B, Liu C, Wang S, Pan M, Wang Y, Wang D, Ye W, Chang L, Zhang W, Song Q, Kirkbride RC, Chen X, Dennis E, Llewellyn DJ, Peterson DG, Thaxton P, Jones DC, Wang Q, Xu X, Zhang H, Wu H, Zhou L, Mei G, Chen S, Tian Y, Xiang D, Li X, Ding J, Zuo Q, Tao L, Liu Y, Li J, Lin Y, Hui Y, Cao Z, Cai C, Zhu X, Jiang Z, Zhou B, Guo W, Li R, Chen ZJ (2015) Sequencing of allotetraploid cotton (Gossypium hirsutum L. acc. TM-1) provides a resource for fiber improvement. Nat Biotechnol 33:531-537

Zhao YL, Wang HM, Shao BX, Chen W, Guo Z, Gong H, Sang X, Wang J, Ye W (2016) SSR-based association mapping of salt tolerance in cotton (Gossypium hirsutum L). Genet Mol Res. https ://doi.org/10.4238/gmr.15027370

Zhu JK (2001) Plant salt tolerance. Trends Plant Sci 6:66-71

Publisher's Note Springer Nature remains neutral with regard to jurisdictional claims in published maps and institutional affiliations. 